TITLE:

\title{
Mechanism of disintegration of charged agglomerates in non- uniform electric field
}

\section{$\operatorname{AUTHOR}(S)$ :}

Shoyama, Mizuki; Matsusaka, Shuji

\section{CITATION:}

Shoyama, Mizuki ... [et al]. Mechanism of disintegration of charged agglomerates in nonuniform electric field. Chemical Engineering Science 2019, 198: 155-164

ISSUE DATE:

2019-04-28

URL:

http://hdl.handle.net/2433/236483

\section{RIGHT:}

(c) 2019. This manuscript version is made available under the CC-BY-NC-ND 4.0 license

http://creativecommons.org/licenses/by-nc-nd/4.0/:; The full-text file will be made open to the public on 28 April 2021 in accordance with publisher's 'Terms and Conditions for Self-Archiving'.; This is not the published version. Please cite only the published version.; この論文は出版社版でありません。引用の際には出版社版をご確認ご利用ください。 
Research Paper for Chemical Engineering Science

\title{
Mechanism of disintegration of charged agglomerates in non-uniform electric field
}

\author{
Mizuki Shoyama, Shuji Matsusaka* \\ Department of Chemical Engineering, Kyoto University, Kyoto 615-8510, Japan
}

\begin{abstract}
The mechanism of disintegration of agglomerates in a non-uniform electric field was studied experimentally and numerically. An external electric field was formed between an upper electrode made of wire mesh and a lower electrode comprising a metal plate. The dielectric particle layers on the lower electrode were charged by induction, and straight-chain agglomerates were formed on the surface of the particle layers by dipole interactions between the particles based on dielectric polarization. In the electric field, the charged agglomerates levitated under the influence of the Coulomb forces, and the charged agglomerates disintegrated with rotation when approaching the upper electrode. The experimental study and analysis of the numerical motion of the agglomerate showed that the charge distribution in the agglomerate generates torques around the centroid of the agglomerate in the non-uniform electric field around the upper electrode, and the centrifugal force acting on the rotating particles causes disintegration of the agglomerates.
\end{abstract}

Keywords: Particle electrification; Electric field; Chain agglomeration; Levitation; Rotation; Disintegration

* Corresponding author. E-mail address: matsu@cheme.kyoto-u.ac.jp (S. Matsusaka). 


\section{Introduction}

Particle charging is a common phenomenon in powder handling. Charged particles are likely to adhere to surfaces due to electrostatic attraction. To remove the adhered particles from the surfaces, fluid drag (Masuda et al., 1994; Gotoh et al., 2015) or vibrations (Theerachaisupakij et al., 2002; Zainuddin et al., 2012; Kobayakawa et al., 2015; Adachi et al., 2017) are often used. Electrostatic forces are also useful because such external forces can control the particle motions without contact (Shoyama and Matsusaka, 2017); furthermore, charged particles with the same polarity can be dispersed by electrostatic repulsion between the particles (Masuda, 2009).

Although semiconducting or dielectric particles polarized in an external electric field form chain agglomerates along the electric field direction (Hollmann, 1950; Pearce, 1954; Itoh et al., 1994; Nakajima and Matsuyama, 2002), the chain agglomerates can undergo disintegration in fluid flow, and the primary particles are dispersed when the electric field is removed. Therefore, the external electric field is useful for controlling particle agglomeration and dispersion in fluid flow (Parthasarathy and Klingenberg, 1996; See and Doi, 1991).

Charges cannot move through ideal dielectric materials; however, actual dielectric materials have a low degree of conductivity. Hence, dielectric particles on an electrode can be charged by induction (Cho, 1964; Wu and Castle, 2003). The particles in the electric field experience interactive forces caused by dielectric polarization and Coulomb forces. Furthermore, if the electric field is non-uniform, gradient forces (Morgan and Green, 1997) also act on the particles.

Control of the motion of charged particles in a non-uniform electric field has been studied for different purposes, such as particle transport, cleaning, classification, and separation. Mazumder et al. (2007) and Calle et al. (2009) reported the transport and removal of particles using several distinctive electrodes with AC voltages having different phases. These methods are applications of the electric curtain (Masuda et al., 1987). In addition, Kawamoto (2008) suggested 
a method of classifying particles based on electrostatic forces. These applications are not limited to solid particles. Dielectrophoresis in a non-uniform electric field has been used to separate oil droplets dispersed in water (Pearce, 1954; Hosseini and Shahavi, 2012). Kacprzyk et al. (2016) reported charge control of droplets sprayed in a non-uniform electric field generated by a mesh electrode.

Shoyama et al. (2018) developed a new system with a vibrating plate electrode and a mesh electrode for continuous particle feed and dispersion, showing that the particles were continuously and effectively dispersed; however, disintegration of the agglomerates was not discussed. The detailed analysis of the motion of charged particles in an electric field and clarification of the mechanism of electrostatic dispersion make it possible to further develop particle dispersion systems without fluid flows.

The goal of this study is to develop methods of controlling the agglomeration, levitation, and dispersion of particles by applying an external electric field. We previously reported that dielectric particles on the lower electrode were charged and levitated in a system comprising a mesh electrode and plate electrode (Shoyama and Matsusaka, 2017). In this study, we analyze the motion of particles using the same electrodes and clarify the mechanism of disintegration of the agglomerates in a non-uniform electric field.

\section{Experimental setup and procedure}

Fig. 1 shows a schematic of the experimental setup used to study the motion of the particles in external electric field. The upper electrode, which was a removable stainless steel wire mesh (material: JIS-SUS304, external dimensions: $60 \times 60 \mathrm{~mm}$, wire diameter: $0.60 \mathrm{~mm}$, opening: 3.63 $\mathrm{mm}$, and distance between centers: $4.23 \mathrm{~mm}$ ), was placed at a distance of $10 \mathrm{~mm}$ from the lower 
electrode, which was a plate electrode (material: JIS-SUS304, external dimensions: $60 \times 60 \mathrm{~mm}$ ). Particle layers of $2 \mathrm{~mm}$ thickness were formed on the lower electrode by gravity feeding. DC +5 $\mathrm{kV}$ voltage that was generated by a power supply (10/10B-HS, TREK, Inc.) was applied to the lower electrode while the upper electrode was grounded. Thus, an upward electric field was generated between the electrodes. The particles that were charged by induction levitated from the particle layers and passed through the upper electrode. The particle motion was recorded at 5000 fps by a high-speed camera (FASTCAM Mini UX100, Photron Ltd.) with a high-magnification zoom lens (VSZ-10100, VS Technology Corporation). A metal halide lamp (LS-M250, Sumita Optical Glass, Inc.) was used as the light source.

Spherical soda-lime glass beads (JIS Z 8901, GBL 100, $D_{\mathrm{p} 50}=100 \mu \mathrm{m}, \rho_{\mathrm{p}}=2300 \mathrm{~kg} / \mathrm{m}^{3}$ ) were used in this study. The particles were dried at $120{ }^{\circ} \mathrm{C}$ for $12 \mathrm{~h}$ and cooled to room temperature in a desiccator before each test. All the experiments were carried out under room conditions, i.e., temperature: $22 \pm 5{ }^{\circ} \mathrm{C}$ and relative humidity: $50 \pm 5 \%$.

\section{Electric field calculation}

The external electric field generated by the upper and lower electrodes was analyzed using commercial software based on the finite element method (COMSOL Multiphysics, AB/COMSOL, Inc.). Fig. 2 shows the simulation model in three-dimensional Cartesian coordinates. The upper electrode was placed in the center of a sufficiently large domain to reduce calculation errors due to boundary conditions. The distance between the electrodes and the electrode configurations for the simulation were the same as those used in the experimental setup. A grid of $0.1 \mathrm{~mm}$, which was $1 / 36$ of the wire mesh opening, was used to analyze the electric field around the wire mesh electrode. The grid size was set to increase towards the edges of the domain to reduce the 
calculation load. The electric potential at the edges of the domain $(x= \pm 300 \mathrm{~mm}, y= \pm 300 \mathrm{~mm}, z$ $= \pm 500 \mathrm{~mm})$ was set to zero as boundary conditions.

Fig. 3(a) shows the calculated electric potential distribution and electric field direction in the $x z$ plane $(-2.12 \leq x \leq 6.35 \mathrm{~mm}$ and $-10.3 \leq z \leq 3.5 \mathrm{~mm})$ at $y=0$. The calculated results in the $y z$ plane at $x=0$ are the same as those in the $x z$ plane because the shape of the mesh opening is square. The electric field between the upper and lower electrodes was almost uniform, except in the vicinity of the upper electrode. Fig. 3(b) shows the details of the electric field around the mesh electrode. The electric field around the upper electrode was directed to the centerlines of the wires. This implies that the motion of the charged particles may be changed along the non-uniform electric field.

\section{Results and discussion}

\subsection{Observation of particle behavior}

Fig. 4 (lower) shows a snapshot of the particles that levitated from the particle layers formed on the lower electrode. Straight-chain agglomerates as well as single particles were observed, as reported by Shoyama and Matsusaka (2017). This phenomenon can be explained as follows: 1) the dielectric particles on the lower electrode are polarized by the upward electric field. 2) Because adhesion forces act between the polarized particles, chain agglomerates are formed. 3) Because the surfaces of the actual particles have a low degree of conductivity, the positive charges move upward by induction; consequently, the particles on the top of the particle layers are also positively charged. 4) The positively charged particles experience upward Coulomb forces in the electric field. 5) Because the Coulomb forces outweigh the adhesion forces, the positively charged 
particles levitate. When the effect of the adhesion forces caused by particle polarization is rather large, chain agglomerates levitate. Fig. 4 (upper) shows a snapshot of the particles around the upper electrode. Straight-chain agglomerates as well as single particles were observed. Motion analysis of the images taken by the high-speed camera indicated that the trajectory and velocity of the agglomerates changed after the particles passed the upper electrode. The detailed motion analysis is described in Section 4.2.

Fig. 5 shows the cumulative number distribution of the agglomerates of different sizes and single particles levitated from the lower electrode. Single particles accounted for more than $60 \%$ of all the levitated species, and the ratio of larger agglomerates was rather small. Because the agglomerates that passed through the upper electrode could disintegrate into primary particles and become dispersed in the air, the number of single particles increased at higher positions.

Fig. 6 shows the probability of disintegration for the agglomerates of different sizes. The height range of observation was $z=0-10 \mathrm{~mm}$ above the upper electrode. The probability is defined as the numerical ratio of agglomerates disintegrated at least once to the total agglomerates. The probability of disintegration increased with an increase in the number of constituent primary particles. This result implies that larger agglomerates disintegrate easily although all the agglomerates may not necessarily disintegrate into primary particles. The probability of disintegration can be controlled by changing the operation conditions.

Fig. 7(a) shows photographs of an agglomerate consisting of three primary particles passing through the upper electrode; the time series photographs were acquired from 2 to $18 \mathrm{~ms}$ at $4 \mathrm{~ms}$ intervals. The agglomerate moved upward, but the distance moved at intervals of 4 ms decreased with an increase in the height. During this movement, the agglomerate gradually changed its state and finally disintegrated. Fig. 7(b) shows enlarged photographs of the same agglomerate, where the photographs were acquired from 6 to $18 \mathrm{~ms}$ at $2 \mathrm{~ms}$ intervals. The agglomerate was in the 
straight-chain structural state at $6 \mathrm{~ms}$. The three primary particles of the agglomerate are denoted as P1, P2, and P3 from top to bottom, respectively. The agglomerate began to rotate counterclockwise remarkably after $6 \mathrm{~ms}$ and P3 separated from the agglomerate and moved upward in the right direction. The agglomerate consisting of the two primary particles continued to rotate counterclockwise; finally, P1 separated from P2. The straight-chain structure observed at $6 \mathrm{~ms}$ changed with the rotation. This implies that the adhesion forces between the particles were not very strong. Dipole interactions based on dielectric polarization may be such adhesion forces. Although the van der Waals force decreases significantly with increasing distance between surfaces, the effect of distance on dipole interactions is rather small. Therefore, even if the contact distance between the particles is somewhat large, the attractive interaction remains operative.

The non-uniform electric field around the wire mesh electrode can affect the rotation and disintegration of the agglomerate. To analyze the motion of each primary particle of the agglomerate in detail, the distances of $\mathrm{P} 1, \mathrm{P} 2$, and $\mathrm{P} 3$ from the centerline of the electrode wire placed on the left side, as shown in Fig. 7(a), were measured.

Fig. 8 shows the time course of the distances of the three primary particles. The experimental lines were almost the same up to $10 \mathrm{~ms}$; however, the line for P3 moved away from the other lines after $10 \mathrm{~ms}$. The lines for P1 and P2 intersected at $15 \mathrm{~ms}$, after which the difference in the distance increased. Therefore, it is considered that the separation of $\mathrm{P} 3$ began at $10 \mathrm{~ms}$ and the separation of P1 began at $15 \mathrm{~ms}$.

\subsection{Method of analysis of motion and trajectory of charged agglomerate}

\subsubsection{Translational motion equation for agglomerate}

When a straight-chain agglomerate, which consists of the same primary particles, in an electric field experiences a drag force $\left(\underline{\mathbf{F}}_{\mathrm{d}}\right)$, a gravitational force $\left(\mathbf{F}_{\mathrm{g}}\right)$, and an electrostatic force 
$\left(\mathbf{F}_{\mathrm{e}}\right)$, the translational motion equation of the agglomerate is expressed as

$$
n m_{\mathrm{p}} \frac{\mathrm{d} \mathbf{v}_{\mathrm{a}}}{\mathrm{d} t}=\mathbf{F}_{\mathrm{d}}+\mathbf{F}_{\mathrm{g}}+\mathbf{F}_{\mathrm{e}}
$$

where $n$ is the number of constituent primary particles in the agglomerate, $m_{\mathrm{p}}$ is the mass of the primary particles, $\mathbf{v}_{\mathbf{a}}$ is the agglomerate velocity, and $t$ is the time. The mass of the particle is given by

$$
m_{\mathrm{p}}=\frac{\pi D_{\mathrm{p}}^{3} \rho_{\mathrm{p}}}{6}
$$

where $D_{\mathrm{p}}$ is the particle diameter and $\rho_{\mathrm{p}}$ is the particle density. For a stationary fluid, $\mathbf{F}_{\mathrm{d}}$ is determined as (Kasper et al., 1985; Niida and Ohtsuka, 1997)

$$
\mathbf{F}_{\mathrm{d}}=3 \pi \mu D_{\mathrm{a}} \mathbf{v}_{\mathrm{a}} \kappa
$$

Here, $\mu$ is the viscosity of the fluid, $D_{\mathrm{a}}$ is the volume equivalent diameter of the agglomerate given by

$$
D_{\mathrm{a}}=n^{\frac{1}{3}} D_{\mathrm{p}}
$$

$\kappa$ is the dynamic shape factor given by

$$
\frac{1}{\kappa}=\frac{1}{\kappa_{\perp}}+\left(\frac{1}{\kappa_{/ /}}-\frac{1}{\kappa_{\perp}}\right) \cos ^{2} \theta_{\mathrm{m}},
$$

where $\theta_{\mathrm{m}}$ is the angle between the agglomerate axis and moving direction, and $\kappa_{\perp}$ and $\kappa_{/ /}$are the dynamic shape factor perpendicular and parallel to the moving direction, respectively, i.e.,

$$
\kappa_{\perp}= \begin{cases}1.00 & (n=1) \\ 1.16 & (n=2) \\ 1.26 & (n=3)\end{cases}
$$

and

$$
\kappa_{/ /}=\left\{\begin{array}{ll}
1.00 & (n=1) \\
1.03 & (n=2) \\
1.07 & (n=3)
\end{array} .\right.
$$


The gravitational force of the agglomerate is given by

$$
\mathbf{F}_{\mathrm{g}}=n m_{\mathrm{p}} \mathbf{g}
$$

where $\mathbf{g}$ is the gravitational acceleration. $\mathbf{F}_{\mathrm{e}}$ is the summation of the Coulomb force in the external electric field $\left(\mathbf{F}_{\text {ex }}\right)$ and the gradient force $\left(\mathbf{F}_{\text {grad }}\right)$, i.e.,

$$
\mathbf{F}_{\mathrm{e}}=\mathbf{F}_{\mathrm{ex}}+\mathbf{F}_{\text {grad }} \text {. }
$$

Here, $\mathbf{F}_{\mathrm{ex}}$ is expressed as

$$
\mathbf{F}_{\mathrm{ex}}=\sum_{i=1}^{n} \mathbf{F}_{\mathrm{q} i}
$$

where $\mathbf{F}_{\mathrm{q} i}$ is the Coulomb force acting on the $i$-th charged particle, given by

$$
\mathbf{F}_{\mathrm{q} i}=q_{i} \mathbf{E}_{\mathrm{ex}},
$$

where $q_{i}$ is the charge of the $i$-th particle and $\mathbf{E}_{\text {ex }}$ is the external electric field. $\mathbf{F}_{\text {grad }}$ can be determined as (Hywel and Green, 1997)

$$
\mathbf{F}_{\text {grad }}=\frac{\pi \varepsilon_{0} \varepsilon_{\mathrm{rf}}\left(\varepsilon_{\mathrm{rp}}-1\right)}{4\left(\varepsilon_{\mathrm{rp}}+2\right)} D_{\mathrm{a}}^{3} \operatorname{grad} \mathbf{E}_{\mathrm{ex}}^{2},
$$

where $\varepsilon_{0}$ is the vacuum permittivity, $\varepsilon_{\mathrm{rf}}\left(\approx 1\right.$ for air) is the relative permittivity of the fluid, and $\varepsilon_{\mathrm{rp}}$ $(\approx 5)$ is the relative permittivity of the particle.

\subsubsection{Rotational motion equation for agglomerate}

When the external forces acting on each primary particle in an agglomerate are not the same, the agglomerate experiences the forces in the circumferential direction as well as in the radial direction.

Fig. 9 shows the rotation models of a chain agglomerate consisting of three primary particles $(n=3)$ and two primary particles $(n=2)$ in an electric field, $\mathbf{E}_{\mathrm{ex}}$. The rotation generates a centrifugal force, $\mathbf{F}_{\mathrm{c}}$, which acts as a separation force for disintegration of the agglomerates.

The rotational motion equation for the agglomerate consists of the torque $\left(\mathbf{T}_{\mathrm{q}}\right)$ caused by the 
difference in the Coulomb force on each primary particle, the torque $\left(\mathbf{T}_{\mathrm{p}}\right)$ caused by the dipole interactions between the primary particles based on dielectric polarization, and the torque $\left(\mathbf{T}_{\mathrm{d}}\right)$ caused by the rotational drag forces of the primary particles; therefore, the rotational motion equation is expressed as

$$
I \frac{d \boldsymbol{\omega}}{d t}=\mathbf{T}_{\mathrm{q}}+\mathbf{T}_{\mathrm{p}}+\mathbf{T}_{\mathrm{d}}
$$

where $I$ is the moment of inertia given by

$$
I=\sum_{i=1}^{n} m_{\mathrm{p}} r_{i}^{2}
$$

where $r_{i}$ is the distance between the centroids of the $i$-th particle and the agglomerate, $\boldsymbol{\omega}$ is the angular velocity, and the magnitude of $\omega$ is given by

$$
\omega=\frac{d \theta}{d t}
$$

where $\theta$ is the angle of the agglomerate axis relative to the vertical.

The angle, $\varphi$, between the agglomerate axis and the electric field direction is defined as

$$
\varphi=\theta-\theta_{\text {ex }}
$$

where $\theta_{\mathrm{ex}}$ is the angle of the external electric field direction at the position of the agglomerate relative to the vertical. Therefore, the torque, $\mathbf{T}_{\mathrm{q}}$, around the agglomerate centroid caused by Coulomb forces is given by

$$
\mathbf{T}_{\mathrm{q}}=\sum_{i=1}^{n}\left(\mathbf{F}_{\mathrm{q} i} \times \mathbf{r}_{i}\right)
$$

where $\mathbf{r}_{i}$ is the position of the $i$-th particle relative to its axis of rotation.

The interaction force, $\mathbf{F}_{\mathrm{p} i j}$, between particles polarized in the electric field was defined by Klingenberg et al. (1989) and Washizu and Jones (1994). Although they considered the effect of an induced field caused by the existence of the particles in the electric field, a simple model was also presented by Klingenberg et al. (1989) and Parthasarathy and Klingenberg (1996). Using the 
latter model to reduce the calculation load, the electrostatic interaction force, $\mathbf{F}_{\mathrm{p} i j}$, between the $i$ th and $j$-th polarized particles is determined as

$$
\mathbf{F}_{\mathrm{p} i j}=F_{0}\left(\frac{D_{\mathrm{p}}}{r_{i j}}\right)^{4}\left\{\left(3 \cos ^{2} \varphi-1\right) \mathbf{e}_{r}+\sin 2 \varphi \mathbf{e}_{\varphi}\right\},
$$

where $r_{i j}$ is the distance between the two particle centroids, $\mathbf{e}_{r}$ and $\mathbf{e}_{\varphi}$ are unit vectors in the radial and circumferential directions of the agglomerate, respectively, and $F_{0}$ is expressed as

$$
F_{0}=\frac{3}{16} \pi \varepsilon_{0} \varepsilon_{\mathrm{rf}} D_{\mathrm{p}}{ }^{2}\left(\frac{\varepsilon_{\mathrm{rp}}-1}{\varepsilon_{\mathrm{rp}}+2}\right)^{2} E_{\mathrm{ex}}^{2} .
$$

The first term on the right-hand side of Eq. (18) represents the electrostatic interaction force between the two particles in the radial direction; thus, the magnitude of the adhesion force between the two particles can be estimated. The second term is the force in the circumferential direction. The force acting on the $i$-th particle due to the other particles, excepting the $i$-th particle, $\mathbf{F}_{\mathrm{p} \varphi i}$ is given by

$$
\mathbf{F}_{\mathrm{p} \varphi i}=\sum_{j=1}^{n} F_{0}\left(\frac{D_{\mathrm{p}}}{r_{i j}}\right)^{4} \sin 2 \varphi \mathbf{e}_{\varphi} \quad(j \neq i) .
$$

Thus, the torque, $\mathbf{T}_{\mathrm{p}}$, caused by the dipole interactions between primary particles polarized in the electric field is given by

$$
\mathbf{T}_{\mathrm{p}}=\sum_{i=1}^{n}\left(\mathbf{F}_{\mathrm{p} \varphi i} \times \mathbf{r}_{i}\right)
$$

In the same manner, the torque, $\mathbf{T}_{\mathrm{d}}$, caused by the fluid drag forces of $i$-th primary particles, $\mathbf{F}_{\mathrm{d} i}$, is given by

$$
\mathbf{T}_{\mathrm{d}}=\sum_{i=1}^{n}\left(\mathbf{F}_{\mathrm{d} i} \times \mathbf{r}_{i}\right)
$$

$\mathbf{F}_{\mathrm{d} i}$ is expressed as a function of the velocity of $i$-th particle, $\mathbf{v}_{\mathrm{p}}$, i.e.,

$$
\mathbf{F}_{\mathrm{d} i}=3 \pi \mu D_{\mathrm{p}} \mathbf{v}_{\mathrm{p} i}
$$

\subsubsection{Calculation method}


Fig. 10 shows the flow chart of the calculation used to determine the motion of the agglomerates. The initial values of the charge, velocity, and position of the agglomerate were set according to the experimental data. The electric fields obtained by numerical simulation (see Section 3) were used for the calculation. The motion analysis of the agglomerate was based on Eqs. (1) and (13). The translational and angular velocities, and the position and axial angle of the agglomerate were obtained numerically using intervals of $0.1 \mathrm{~ms}$. Thus, the trajectories of the agglomerate were obtained as a function of the initial charge of the primary particles.

\subsection{Experimental and analytical results for motion and trajectory of charged agglomerate}

\subsubsection{Translational motion}

For detailed analysis of the motion of a charged agglomerate, we selected a straight-chain agglomerate observed by the high-speed camera. The focus depth of the lens used was less than $20 \mu \mathrm{m}$.

Fig. 11 shows the vertical position of the agglomerate observed at $0.2 \mathrm{~ms}$ intervals. This agglomerate is the same as that shown in Fig. 7. The agglomerate was located under the wire mesh electrode at times of $2 \mathrm{~ms}$ or less. The solid line in this figure indicates the calculated position of the particle obtained by fitting. The experimental values agreed well with the calculated line. The fitting parameter was the initial charge of agglomerate, and the value was $0.165 \mathrm{pC}$.

Fig. 12 shows the trajectory and disintegration of the agglomerate. The experimental data correspond to the position of $\mathrm{P} 2$ at 2 ms intervals. The agglomerate consisting of $\mathrm{P} 1, \mathrm{P} 2$, and $\mathrm{P} 3$ levitated from the left side of the center of the opening of the wire mesh electrode. During this levitation, $\mathrm{P} 3$ and $\mathrm{P} 1$ separated from $\mathrm{P} 2$. After reaching the highest position at $z=3.3 \mathrm{~mm}, \mathrm{P} 2$ moved in the lower left direction. The calculated trajectory (a solid line) is also shown in the same 
figure. In this calculation, we considered the variation of the number of constituent primary particles according to the particle separation, as well as the charge distribution in the agglomerate caused by electrostatic induction in the external electric field. Generation of the charge distribution in the agglomerate can be explained as follows: 1) dielectric particles form chain agglomerates owing to the dipole interactions based on dielectric polarization. 2) Because the surfaces of the actual particles have a low degree of conductivity, the particles on the lower electrode are charged by induction and levitate. 3) During levitation in the external electric field, the charges on the chain agglomerates move by electrostatic induction; thus, the charge of the upper primary particle becomes larger. Assuming that the charge distribution is $q_{1}: q_{2}: q_{3}=3: 2: 1$ for $\mathrm{P} 1, \mathrm{P} 2$, and $\mathrm{P} 3$, respectively, the experimental and calculated trajectory data are in good agreement. The above assumption is further discussed in the next section.

\subsubsection{Rotational motion}

Fig. 13 shows the experimental data for the rotation of the agglomerate, i.e., the variation of the angle $\theta$ between the agglomerate axis and the vertical. The agglomerate axis is defined as a straight line connecting two centroids of the primary particles at the ends of the agglomerate; thus, the value of $\theta$ for $n=3$ differs somewhat from that for $n=2$. The agglomerate, which is the same as that discussed in Section 4.3.1, began to rotate counterclockwise after $5 \mathrm{~ms}$. The $\theta$ value for separation of the first primary particle at $10 \mathrm{~ms}$ was approximately $\pi / 2 \mathrm{rad}$, and that for separation of the second primary particle at $15 \mathrm{~ms}$ was approximately $3 \pi / 2 \mathrm{rad}$. The inclination of the experimental curve, namely the angular velocity, tended to increase with time. This implies that the agglomerate rotates with angular acceleration.

Fig. 14 shows the effect of the particle charge distribution on the rotation of the agglomerate. 
The experimental data are indicated by the solid line, which is the same as that shown in Fig. 13. The calculated results from the rotational motion equation are indicated by broken lines. The total charge of the agglomerate used for the calculation was $0.165 \mathrm{pC}$, as mentioned above. The local electric field strength, $E_{\text {ex }}$, was $16.5 \mathrm{kV} / \mathrm{m}$. When there was no charge distribution in the agglomerate, i.e., $q_{1}=q_{2}=q_{3}$, the variation of the calculated rotation angle with time was extremely small. Assuming that $q_{1}: q_{2}: q_{3}=3: 2: 1$, the rotation angle increased, and the consistency between the experimental results and the calculated data was improved. Although there was still some difference between the experimental and calculated results, this is probably caused by the deviation of the straight-chain structure during the rotation.

\subsection{Mechanism of agglomerate rotation}

Fig. 15 shows the torques acting on two particles with different $\varphi$ values. Here, it is assumed that the charge of the upper particle, $q_{1}$, is larger than the charge, $q_{2}$, for the lower particle. The larger charge generates a stronger Coulomb force; thus, the particles experience a torque $\left(\mathbf{T}_{\mathrm{q}}\right)$ around the centroid. This torque depends on the angle $\varphi$ between the particle axis and the electric field direction. $\mathbf{T}_{\mathrm{q}}$ acts clockwise for $0<\varphi<\pi$ (Fig. 15(b), (c), and (d)), counterclockwise for $-\pi$ $<\varphi<0$ (Fig. 15 (f), (g), and (h)), and $\mathbf{T}_{\mathrm{q}}=0$ for $\varphi=0$ and $\varphi= \pm \pi$, (Fig. 15 (a) and (e)). In addition, the particles experience a torque $\left(\mathbf{T}_{\mathrm{p}}\right)$ caused by the dipole interactions based on dielectric polarization in the electric field. As $\mathbf{T}_{\mathrm{p}}$ causes the particle axis to align with the electric field direction, it acts clockwise for $0<\varphi<\pi / 2$ and $-\pi<\varphi<-\pi / 2$ (Fig. 15 (b) and (f)), counterclockwise for $\pi / 2<\varphi<\pi$ and $-\pi / 2<\varphi<0$ (Fig. 15 (d) and (h)), and $\mathbf{T}_{\mathrm{p}}=0$ for $\varphi=0, \varphi= \pm \pi / 2$, and $\varphi= \pm \pi$ (Fig. 15 (a), (c), (e), and (g)).

Fig. 16 shows a comparison between the $T_{\mathrm{q}}$ and $T_{\mathrm{p}}$ values calculated using Eqs. (17) and (21). The parameters for the calculation are based on the agglomerate in Fig. 14, i.e., $q_{1}=0.083 \mathrm{pC}, q_{2}$ 
$=0.055 \mathrm{pC}$, and $E_{\mathrm{ex}}=16.5 \mathrm{kV} / \mathrm{m}$. The positive and negative values of the torques correspond to the clockwise and counterclockwise directions, respectively. The calculated results show that $T_{\mathrm{q}}$ is positive for $-\pi<\varphi<0$ and negative for $0<\varphi<\pi$. The maximum absolute value of $T_{\mathrm{q}}$ was 22.7 $\mathrm{fN} \cdot \mathrm{m}$ and its cycle was $2 \pi$. Meanwhile, those of $T_{\mathrm{p}}$ were $0.4 \mathrm{fN} \cdot \mathrm{m}$ and $\pi$, respectively. The maximum absolute value of $T_{\mathrm{q}}$ was much larger than that of $T_{\mathrm{p}}$; therefore, the rotation of the agglomerate was found to be controlled by $T_{\mathrm{q}}$. If $q_{1}=q_{2}$ or the difference is quite small, $T_{\mathrm{q}}$ will be smaller than $T_{\mathrm{p}}$, making rotation of the agglomerate difficult.

Fig. 17 shows the variation of the angle $\varphi$ between the agglomerate axis and the local electric field direction. The value as a function of time is based on motion analysis of the agglomerate with $n=3$ and the calculated electric field data. The value of $\varphi$ is basically negative at $t<8 \mathrm{ms,}$ where the state of the agglomerate is the same as that in Fig. 15(h), and thus the agglomerate experiences counterclockwise torques. After rotating counterclockwise, the value of $\varphi$ changes from negative to positive and increases with time. Based on the state of the agglomerate at $t>8$ ms, which corresponds to that in Fig. 15(b), the clockwise torques lower the counterclockwise rotation.

\subsection{Mechanism of disintegration of the agglomerates}

Rotation of the agglomerate generates centrifugal forces that can separate the primary particles from the agglomerate. The magnitude of the centrifugal force, $\left|F_{\mathrm{c}}\right|$, acting on the primary particles is given by

$$
\left|F_{\mathrm{c}}\right|=m_{\mathrm{p}} r \omega^{2}
$$

Fig. 18 shows the analytical data for the temporal variation of the centrifugal force, $\left|F_{\mathrm{c}}\right|$, and adhesion force, $\left|F_{\mathrm{a}}\right|$, acting on the primary particles at the end of the agglomerate. The values of $\left|F_{\mathrm{c}}\right|$ were calculated by using the angular velocities obtained from differentiation of the $\theta$ values. 
The values of $\left|F_{\mathrm{a}}\right|$ caused by the particle polarization were calculated as the first term on the righthand side of Eq. (18). The values of $E_{\mathrm{ex}}$ and $\varphi$ in this equation were determined by considering the time courses of the state and position of the agglomerate and the results. Consequently, it was found that $\left|F_{\mathrm{a}}\right|$ was initially very high but decreased with time; however, $\left|F_{\mathrm{c}}\right|$ became larger with time for both $n=3$ and $n=2$. Particle separation was initiated at 10 and $15 \mathrm{~ms}$ when $\left|F_{\mathrm{c}}\right|>\left|F_{\mathrm{a}}\right|$ was satisfied. Therefore, we can conclude that the centrifugal force plays a significant role in the disintegration of the agglomerate rotating in the non-uniform electric field. Van der Waals forces might act somewhat as the adhesion force. Although it is difficult to estimate the accurate value, if it is assumed that the primary particles have nano-roughness on the surfaces, the adhesion force is as small as $10 \mathrm{nN}$. Therefore, there are no contradictions in the above explanation for the rotational separation.

\section{Conclusion}

It was empirically demonstrated that the dielectric particle layers on the lower electrode were charged by induction, and the straight-chain agglomerates that formed on the surface of the particle layers levitated in the uniform electric field. The agglomerate rotated and disintegrated in the non-uniform electric field around the upper wire mesh electrode. To clarify the mechanism of rotation and disintegration, the particle trajectories obtained experimentally before and after the disintegration were analyzed and compared with those obtained numerically using the motion equation and considering the simulated electric field. The analysis showed that the primary particles constituting the agglomerate have different charges caused by electrostatic induction; i.e., a charge distribution develops in the chain agglomerate. When the agglomerate is in a nonuniform electric field, these primary particles experience different Coulomb forces; consequently, 
an electrostatic torque is generated around the agglomerate centroid. Meanwhile, dipole interactions between the primary particles based on the dielectric polarization also generate a torque; however, the magnitude of this torque is much smaller than that mentioned above. Therefore, rotation of the agglomerate is controlled by the torque based on the charge distribution in the agglomerate. Furthermore, the rotation generates a centrifugal force, which causes disintegration of the agglomerate.

\section{Acknowledgement}

This research was supported by JSPS KAKENHI Grant Number JP17H03442.

\section{Nomenclature}

$D_{\mathrm{a}} \quad$ volume equivalent diameter of agglomerate (m)

$D_{\mathrm{p}} \quad$ particle diameter (m)

$\mathbf{E}_{\mathrm{ex}} \quad$ external electric field $(\mathrm{V} / \mathrm{m})$

e unit vector $(-)$

$\mathbf{F}_{\mathrm{a}} \quad$ adhesion force $(\mathrm{N})$

$\mathbf{F}_{\mathrm{c}} \quad$ centrifugal force $(\mathrm{N})$

$\mathbf{F}_{\mathrm{d}} \quad$ drag force $(\mathrm{N})$

$\mathbf{F}_{\mathrm{e}} \quad$ electrostatic force $=\mathbf{F}_{\mathrm{ex}}+\mathbf{F}_{\text {grad }}(\mathrm{N})$

$\mathbf{F}_{\mathrm{ex}} \quad$ Coulomb force acting on agglomerate in external electric field (N)

F $_{\mathrm{g}} \quad$ gravitational force $(\mathrm{N})$

F $_{\text {grad }} \quad$ gradient force $(\mathrm{N})$

$\mathbf{F}_{\mathrm{p} i j} \quad$ interaction force between $i$-th and $j$-th particles polarized in the external electric field $(\mathrm{N})$ 
$\mathbf{F}_{\mathrm{q}} \quad$ Coulomb force acting on primary particle $(\mathrm{N})$

g gravitational acceleration $\left(\mathrm{m} / \mathrm{s}^{2}\right)$

I moment of inertia $\left(\mathrm{kg} \cdot \mathrm{m}^{2}\right)$

$m_{\mathrm{p}} \quad$ mass of primary particle $(\mathrm{kg})$

number of constituent primary particles in agglomerate $(-)$

$p_{\mathrm{d}}$

probability of disintegration of agglomerate (-)

$q$

charge of primary particle (C)

$\mathbf{r}$

particle position relative to its axis of rotation $(\mathrm{m})$

$r_{i j}$

$\mathbf{T}_{\mathrm{q}} \quad$ torque caused by difference in Coulomb force of each primary particle $(\mathrm{N} \cdot \mathrm{m})$

$\mathbf{T}_{\mathrm{p}} \quad$ torque caused by interaction between polarized primary particles $(\mathrm{N} \cdot \mathrm{m})$

$\mathbf{T}_{\mathrm{d}} \quad$ torque caused by rotational drag force $(\mathrm{N} \cdot \mathrm{m})$

$t \quad$ time (s)

$\mathbf{v}_{\mathrm{a}} \quad$ velocity of agglomerate $(\mathrm{m} / \mathrm{s})$

$\mathbf{v}_{\mathrm{p}} \quad$ velocity of particle $(\mathrm{m} / \mathrm{s})$

horizontal coordinate $(\mathrm{m})$

$x_{\mathrm{p}} \quad$ particle position in $x$-direction (m)

$y$

horizontal coordinate perpendicular to $x$ coordinate (m)

$Z$

vertical coordinate $(\mathrm{m})$

$z_{\mathrm{p}} \quad$ particle position in $z$-direction $(\mathrm{m})$

$\varepsilon_{0}$

vacuum permittivity $=8.85 \times 10^{-12}(\mathrm{~F} / \mathrm{m})$

$\varepsilon_{\mathrm{rf}}$

relative permittivity of fluid (-)

$\varepsilon_{\mathrm{rp}} \quad$ relative permittivity of particle (-)

$\theta \quad$ angle of agglomerate axis from vertical (rad) 


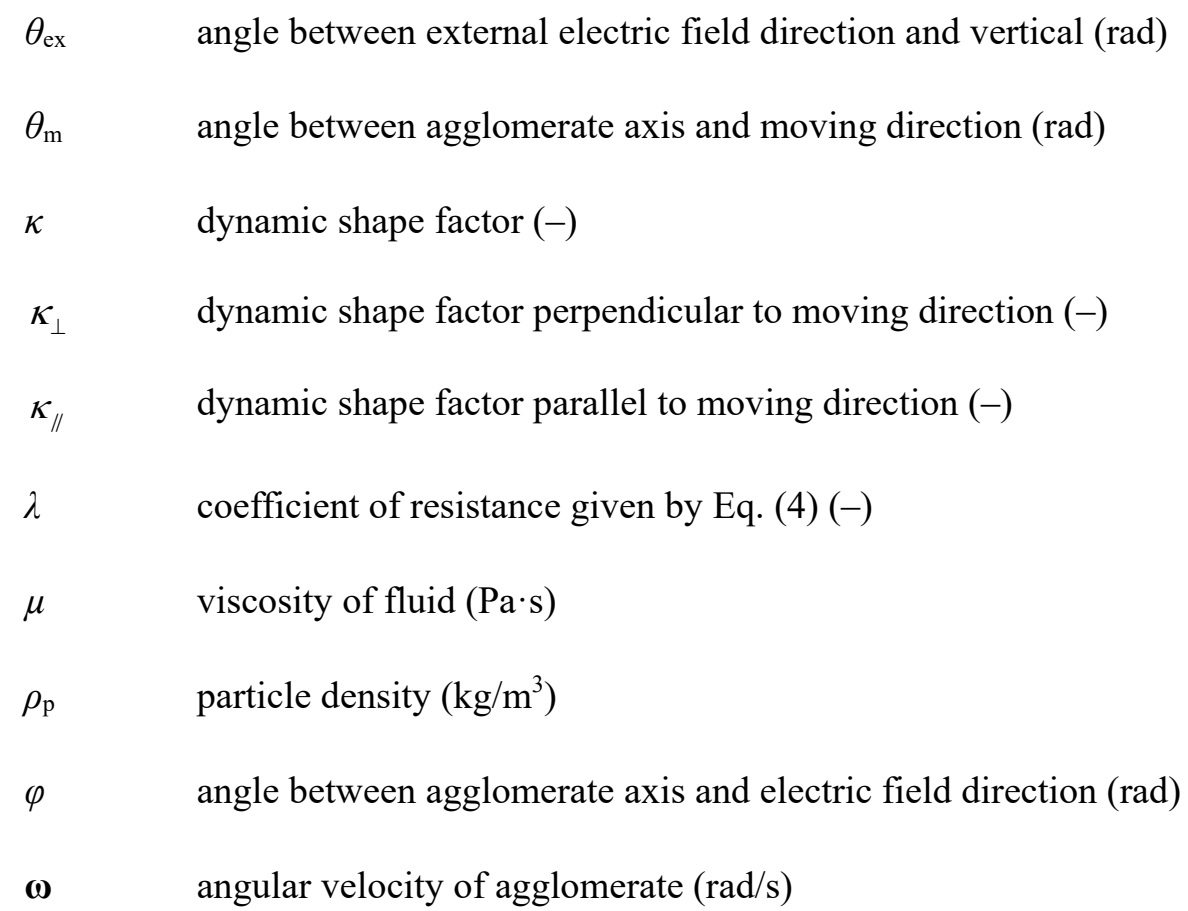

subscripts

$i \quad$ index for primary particle

$r \quad$ radial direction

$\varphi \quad$ circumferential direction

$x \quad$ horizontal direction

$z \quad$ vertical direction

\section{References}

Adachi M., Hamazawa K., Mimuro Y., Kawamoto H., 2017. Vibration transport system for lunar and Martian regolith using dielectric elastomer actuator. J. Electrostat. 89, 88-98.

Calle, C. I., Buhler, C. R., McFall, J. L., Snyder, S. J. 2009. Particle removal by electrostatic and dielectrophoretic forces for dust control during lunar exploration missions. J. Electrost. 
$67,89-92$.

Cho, A. Y. H., 1964. Contact charging of micron-sized particles in intense electric fields. J. Appl. Phys. 35, 2561-2564.

Gotoh, K., Mizutani, K., Tsubota, Y., Oshitani, J., Yoshida, M., Inenaga K., 2015. Enhancement of particle removal performance of high-speed air jet by setting obstacle in jet flow. Particul. Sci. Technol. 33, 567-571.

Hollmann, H. E., 1950. Semiconductive Colloidal Suspensions with Non-Linear Properties. J. Appl. Phys. 21, 402-413.

Hosseini, M., Shahavi, M. H., 2012. Electrostatic enhancement of coalescence of oil droplets (in nanometer scale) in water emulsion. Chinese J. Chem. Eng. 20, 654-658.

Hywel, M., Green, N. G., 1997. Dielectrophoretic manipulation of rod-shaped viral particles. J. Electrost. 42, 279-293.

Itoh, T., Masuda, S., Gomi, F., 1994. Electrostatic orientation of ceramic short fibers in liquid. J. Electrost. 32, 71-89.

Kacprzyk, R., Lewandowski M., 2016. Post-dispersion electrification of droplets. J. Electrost. 79, $33-37$.

Kasper, G., Niida, T., Yang, M., 1985. Measurements of viscous drag on cylinders and chains of spheres with aspect ratios between 2 and 50. J. Aerosol Sci. 16, 535-556.

Kawamoto, H., 2008. Some Techniques on Electrostatic Separation of Particle Size Utilizing Electrostatic Traveling-wave Field. J. Electrost. 66, 220-228.

Klingenberg, D. J., Swol, F. V., Zukoski, C. F., 1989. Dynamic simulation of electrorheological suspensions. J. Chem. Phys. 91, 7888-7895.

Kobayakawa, M., Kiriyama, S., Yasuda, M., Matsusaka, S., 2015. Microscopic analysis of particle detachment from an obliquely oscillating plate. Chem. Eng. Sci. 123, 388-394.

Masuda H., 2009. Dry dispersion of fine particles in gaseous phase. Adv. Powder Technol. 20, 
$113-122$.

Masuda, H., Gotoh, K., Fukada, H., Banba, Y., 1994. The removal of particles from flat surfaces using a high-speed air jet. Adv. Powder Technol. 5, 205-217.

Masuda, S., Washizu, M., Iwadare, M., 1987. Separation of small particles suspended in liquid by nonuniform traveling field. IEEE T. Ind. Appl. 3, 474-480.

Mazumder, M. K., Sharma, R., Biris, A. S., Zhang, J., Calle C., Zahn M., 2007. Self-cleaning transparent dust shields for protecting solar panels and other devices. Particl. Sci. Technol. $25,5-20$.

Morgan, H., Green, N., 1997. Dielectrophoretic manipulation of rod-shaped viral particles. J. Electrost. 42, 279-293.

Nakajima, Y., Matsuyama, T., 2002. Electrostatic field and force calculation for a chain of identical dielectric spheres aligned parallel to uniformly applied electric field. J. Electrost. $55,203-221$.

Niida, T., Ohtsuka, S., 1997. Dynamic Shape Factors of Regular Shaped Agglomerates and Estimation Based on Agglomerate Symmetry-For Rectangular parallelepiped, V-and Wshaped, Hexagonal and H-shaped Agglomerates-[Translated]. KONA Powder Part. J. 15, $202-211$.

Parthasarathy, M., Klingenberg, D. J., 1996. Electrorheology: mechanisms and models. Mater. Sci. Eng. R17, 57-103.

Pearce, C. A. R., 1954. The mechanism of the resolution of water-in-oil emulsions by electrical treatment. Brit. J. Appl. Phys. 5, 136.

See, H., Doi M., 1991. Aggregation kinetics in electro-rheological fluids. J. Phys. Soc. Jpn. 60, $2778-2782$.

Shoyama, M., Matsusaka S., 2017. Electric charging of dielectric particle layers and levitation of particles in a strong electric field. Kagaku Kogaku Ronbun. 43, 319-326. 
Shoyama, M., Kawata, T., Yasuda, M., Matsusaka S., 2018. Particle electrification and levitation in a continuous particle feed and dispersion system with vibration and external electric fields. Adv. Powder Technol. 29, 1960-1967.

Theerachaisupakij, W., Matsusaka, S., Kataoka, M., Masuda, H., 2002. Effects of wall vibration on particle deposition and reentrainment in aerosol flow. Adv. Powder Technol. 13, 287300.

Washizu, M., Jones, T. B., 1994. Dielectrophoretic interaction of two spherical particles calculated by equivalent multipole-moment method. Conference Record of the 1994 IEEE, 2, 14831490.

Wu, Y., Castle G. S. P., Inculet I. I., Petigny S., Swei G. S., 2003. Induction charge on freely levitating particles. Powder technol., 135, 59-64.

Zainuddin I., Yasuda M., Horio T., Matsusaka S., 2012. Experimental study on powder flowability using vibration shear tube method. Part. Part. Syst. Char. 29, 8-15. 


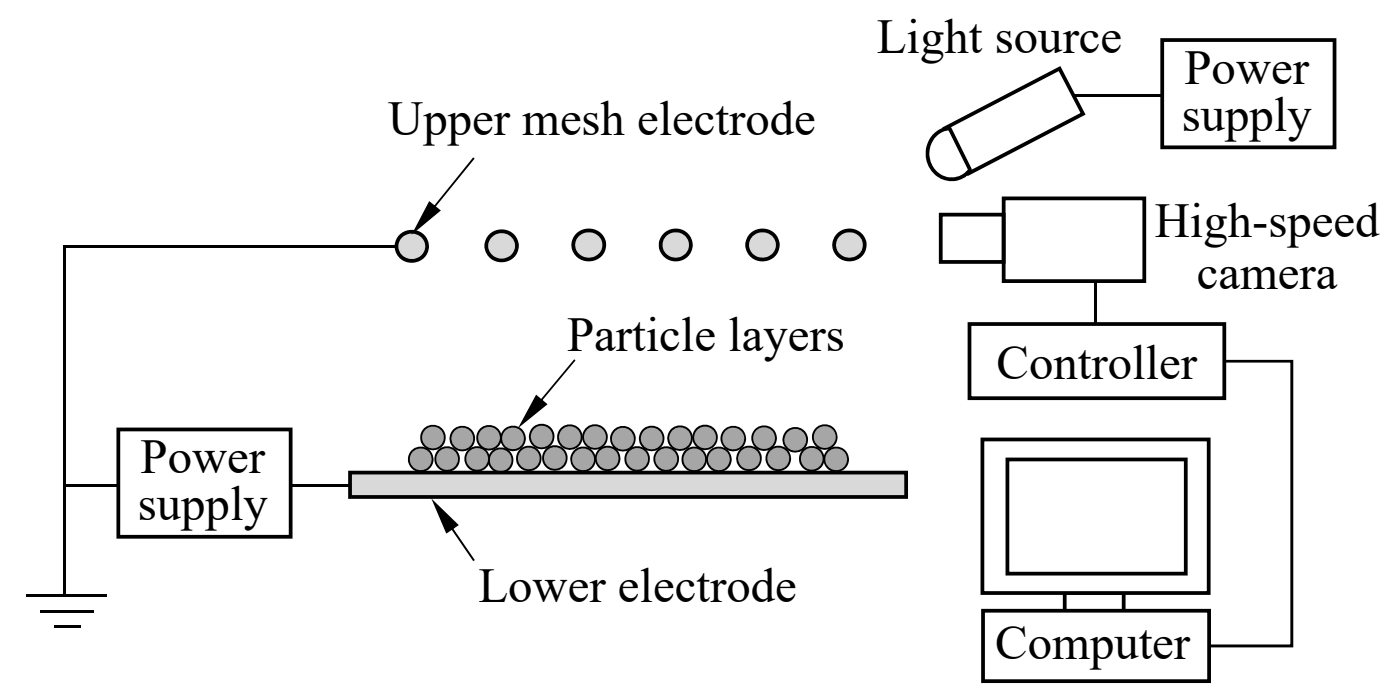

Fig. 1. Schematic of the experimental setup. 


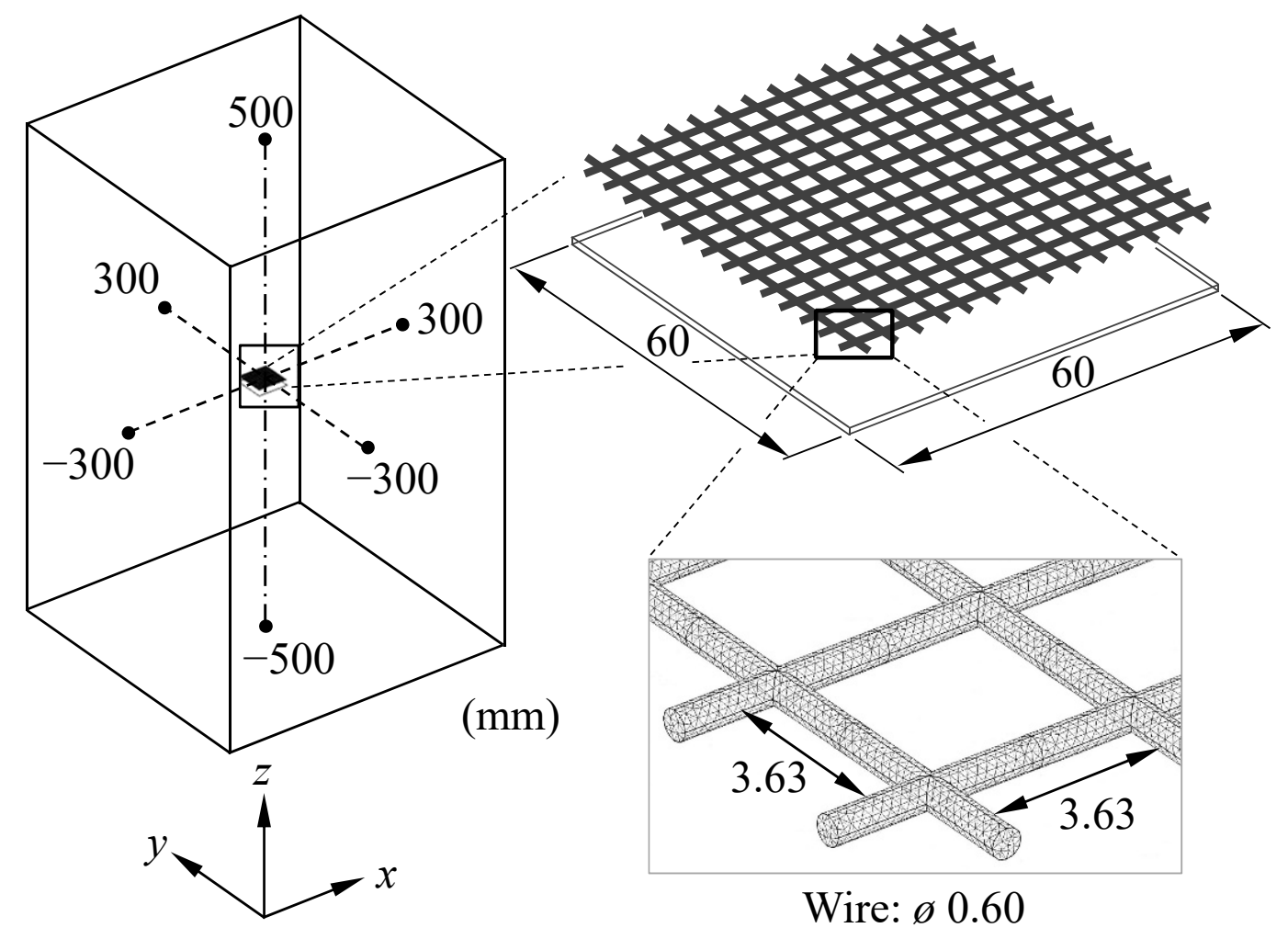

Fig. 2. Simulation model for calculating external electric field. 


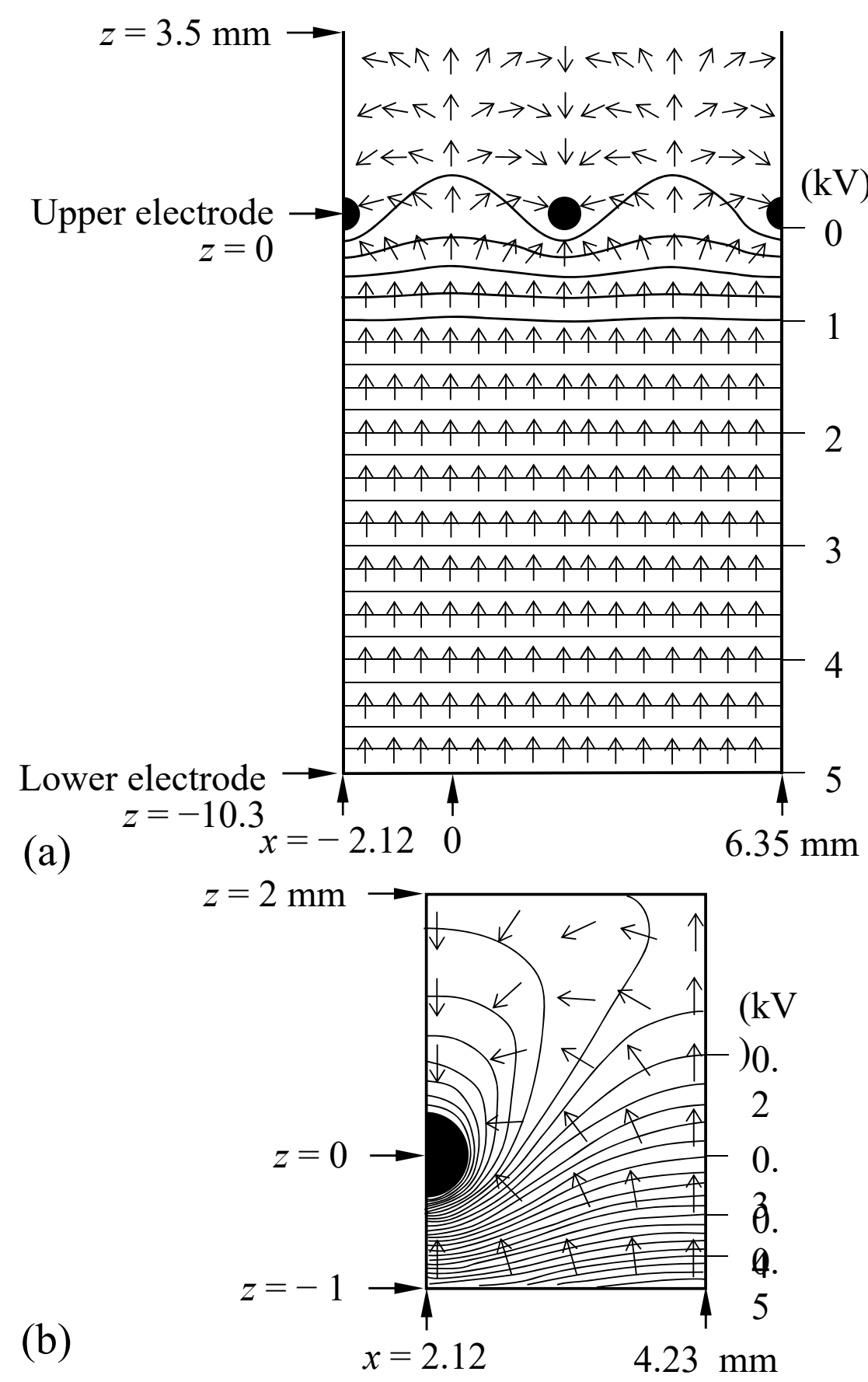

Fig. 3. Calculated electric potential distribution and electric field direction at $y=0$ : (a) $-2.12 \leq x \leq 6.35 \mathrm{~mm}$ and $-10.3 \leq z \leq 3.5 \mathrm{~mm}$; and (b) $2.12 \leq x \leq 4.23 \mathrm{~mm}$ and $-1 \leq z \leq 2 \mathrm{~mm}$. 


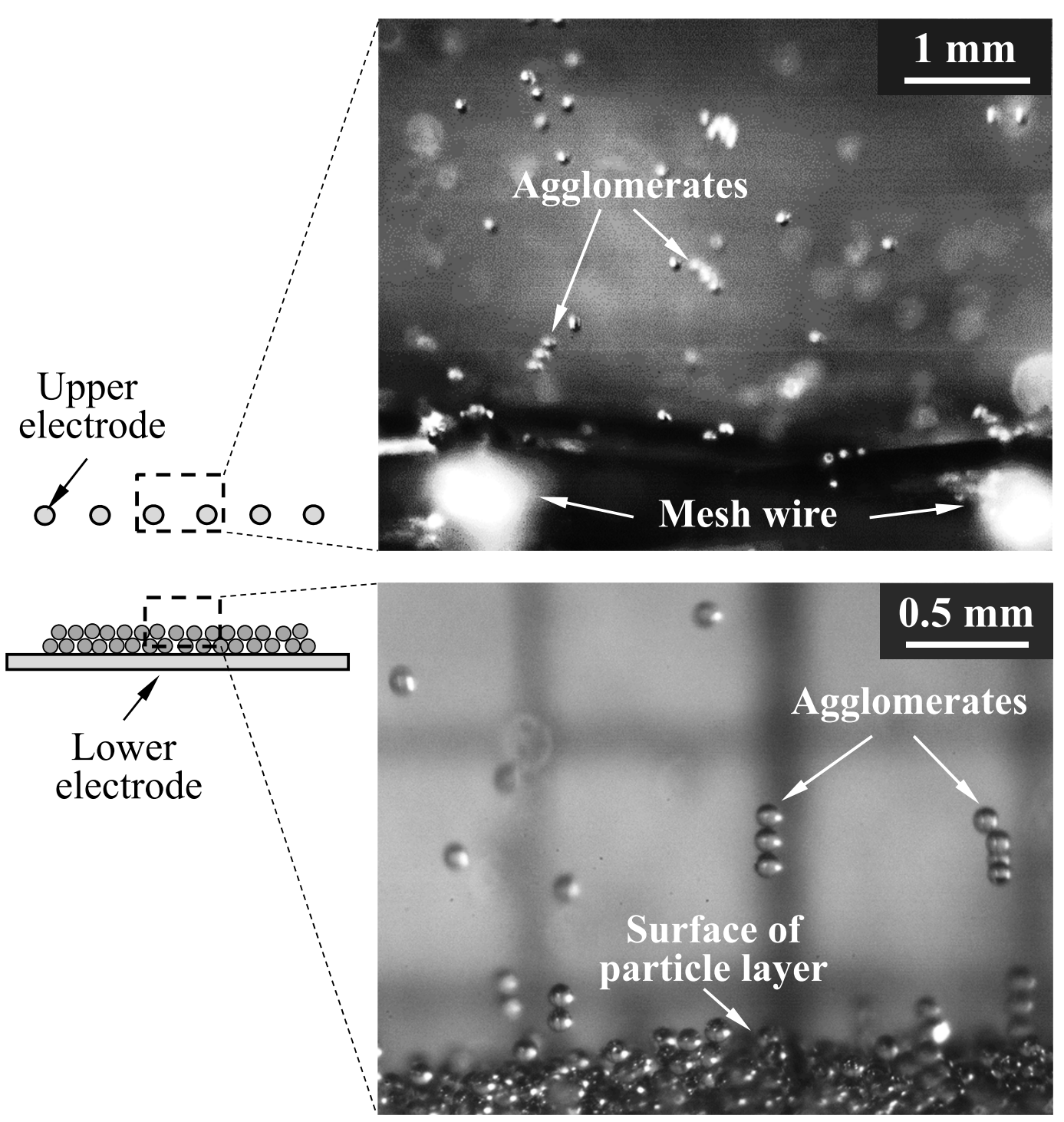

Fig. 4. Snapshots of particles around the upper electrode and particle layers on the lower electrode. 


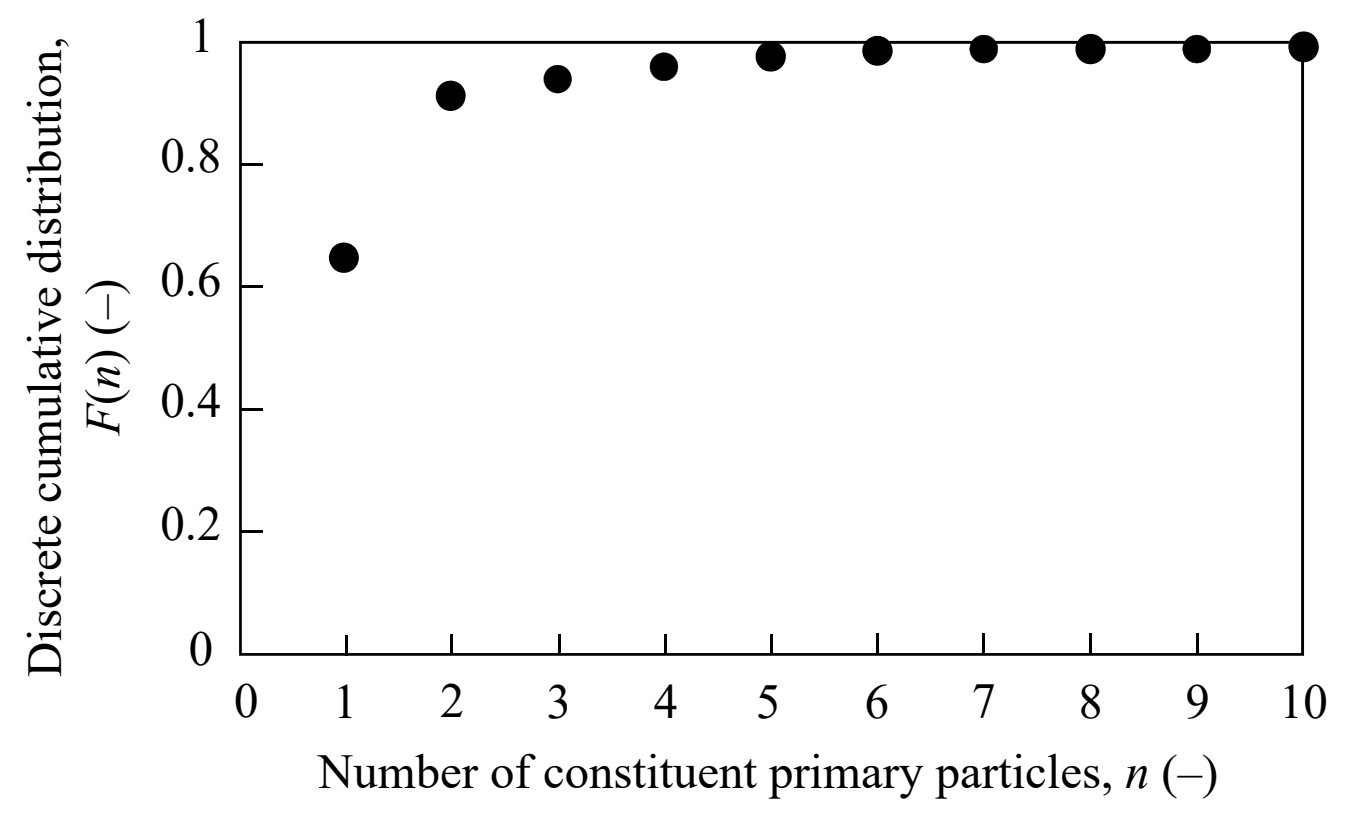

Fig. 5. Cumulative number distribution of agglomerates of different sizes and single particles levitated from the lower electrode. 


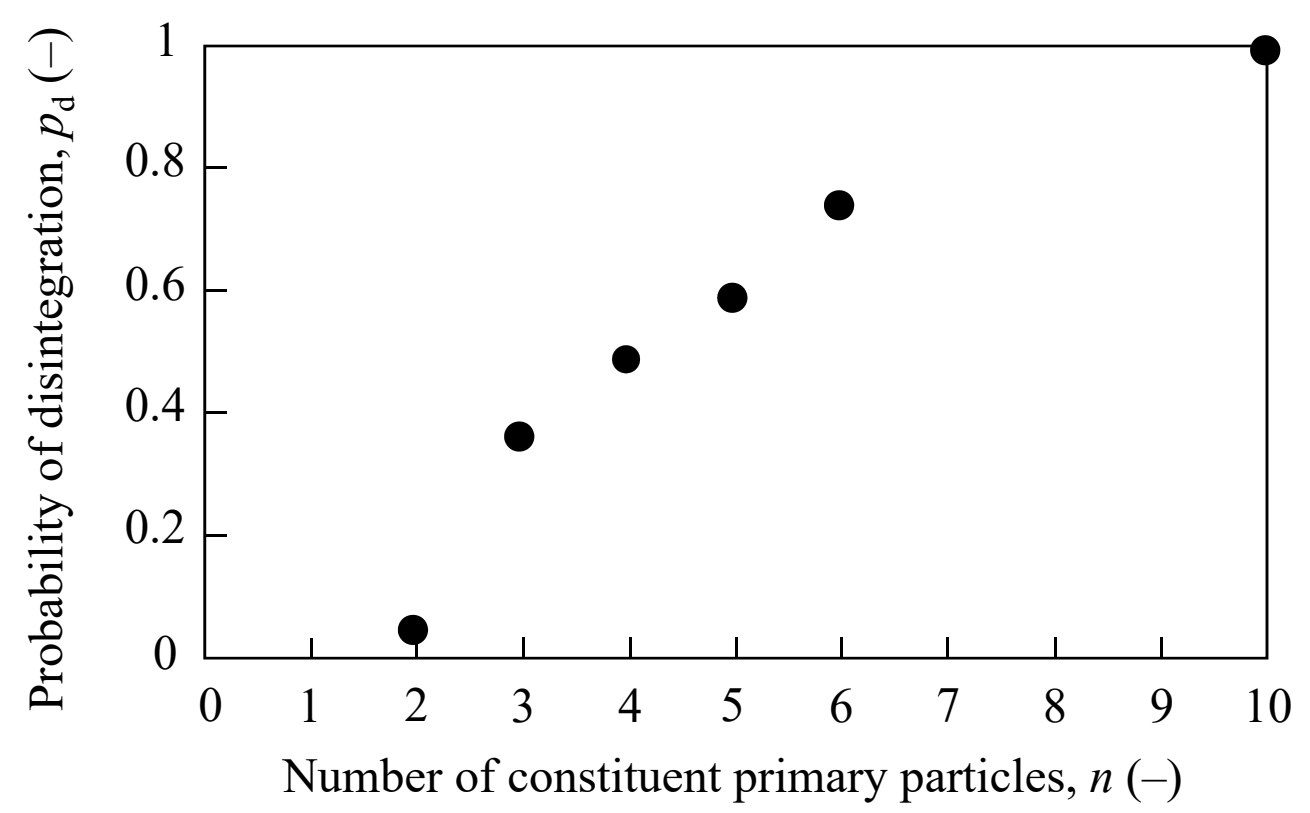

Fig. 6. Probability of disintegration of agglomerates of different sizes. 
(a)

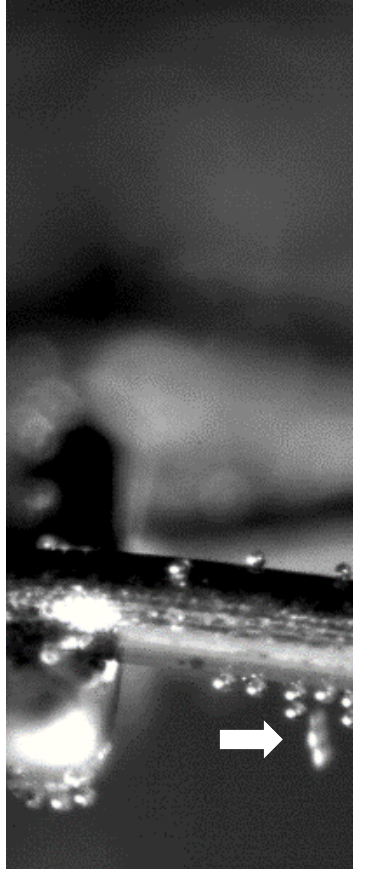

$2 \mathrm{~ms}$

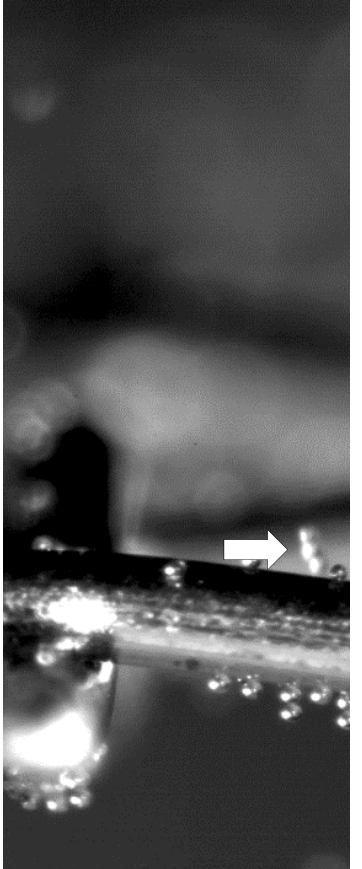

$6 \mathrm{~ms}$

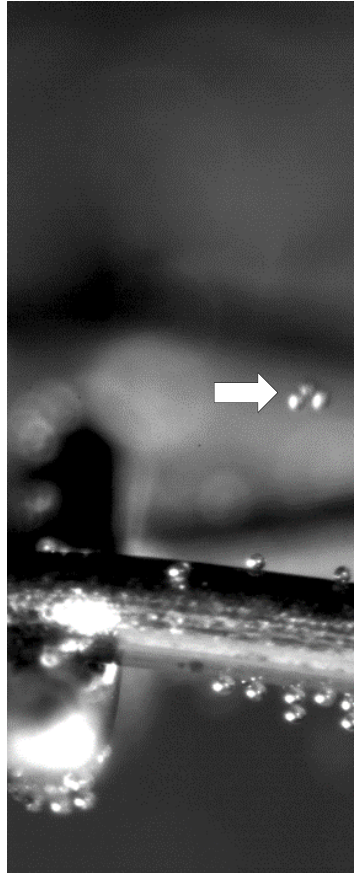

$10 \mathrm{~ms}$

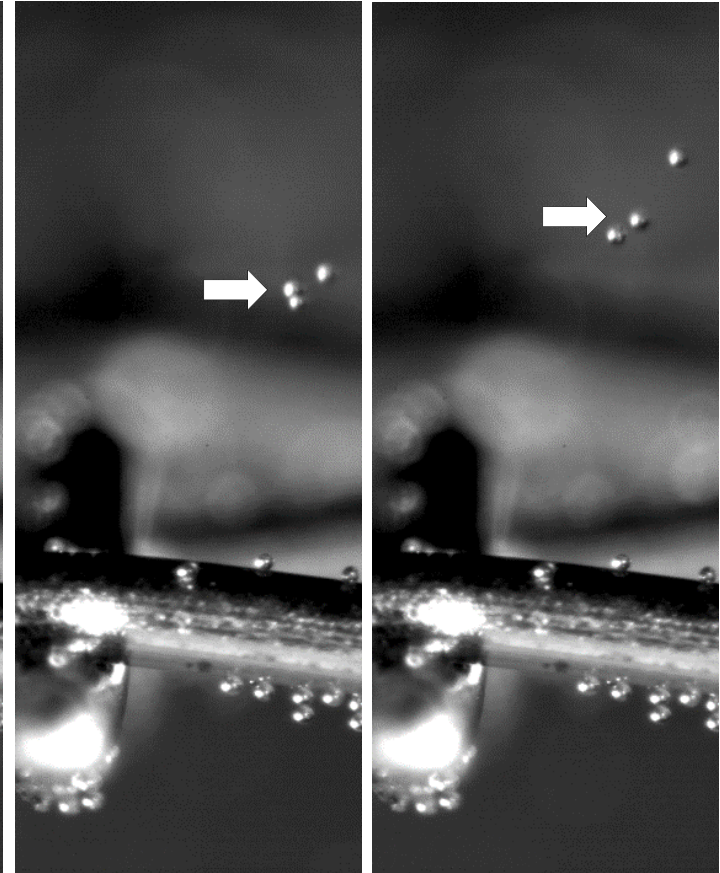

$14 \mathrm{~ms}$
$1 \mathrm{~mm}$

$18 \mathrm{~ms}$

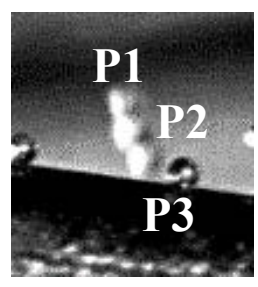

(b)

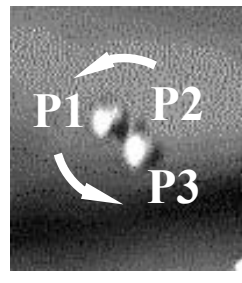

$8 \mathrm{~ms}$

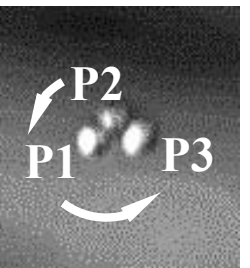

$10 \mathrm{~ms}$

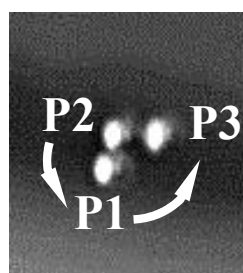

$12 \mathrm{~ms}$

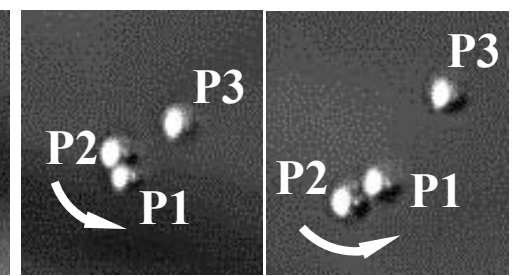

$14 \mathrm{~ms}$

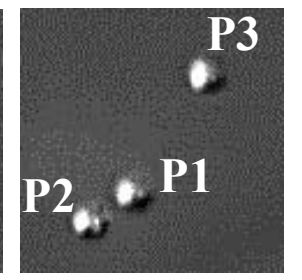

$0.5 \mathrm{~mm}$

$18 \mathrm{~ms}$

Fig. 7. Motion and disintegration of agglomerate passing through the upper electrode:

(a) fixed-point photographs and (b) enlarged photographs of the agglomerate. 


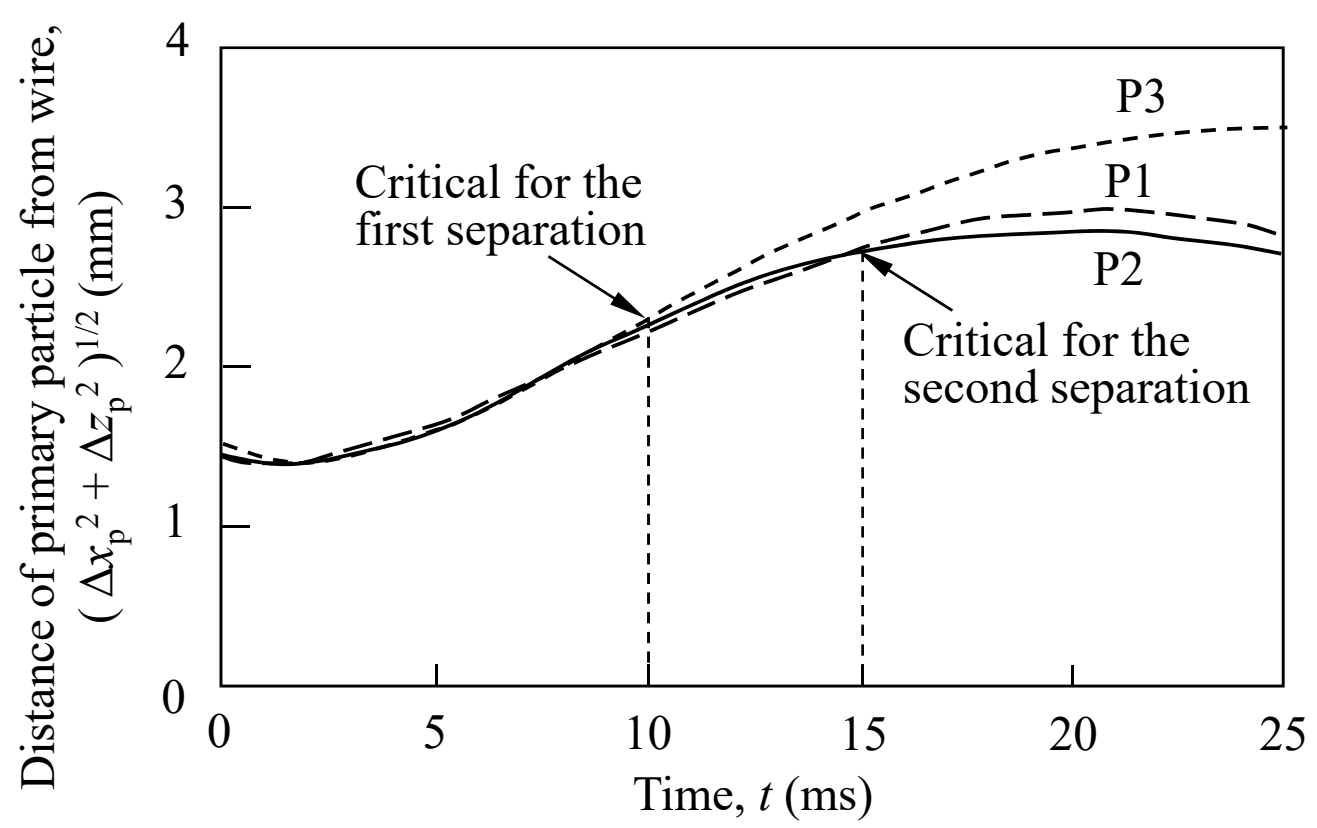

Fig.8. Time course of the distance of the three primary particles from the wire. 
(a) $n=3$

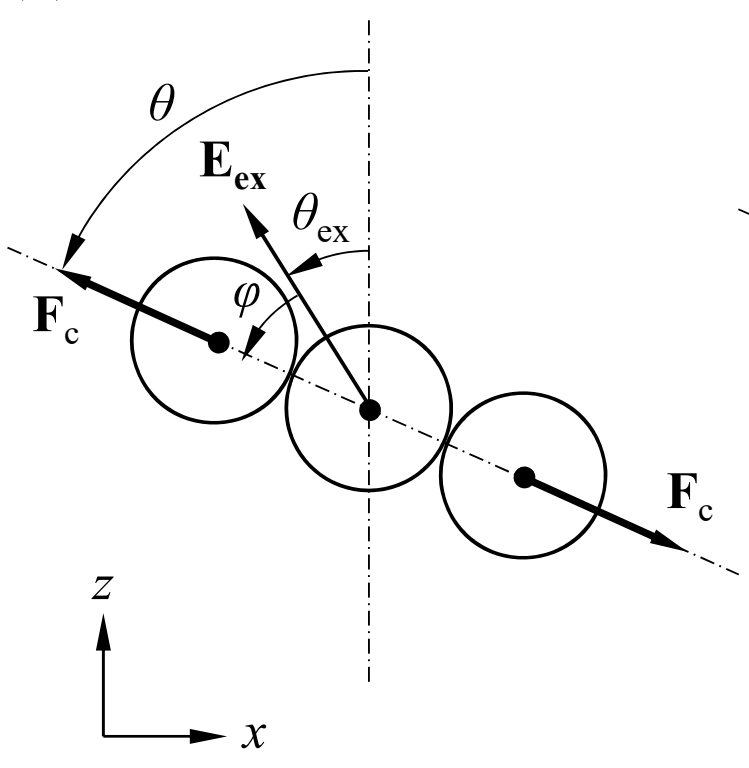

(b) $n=2$

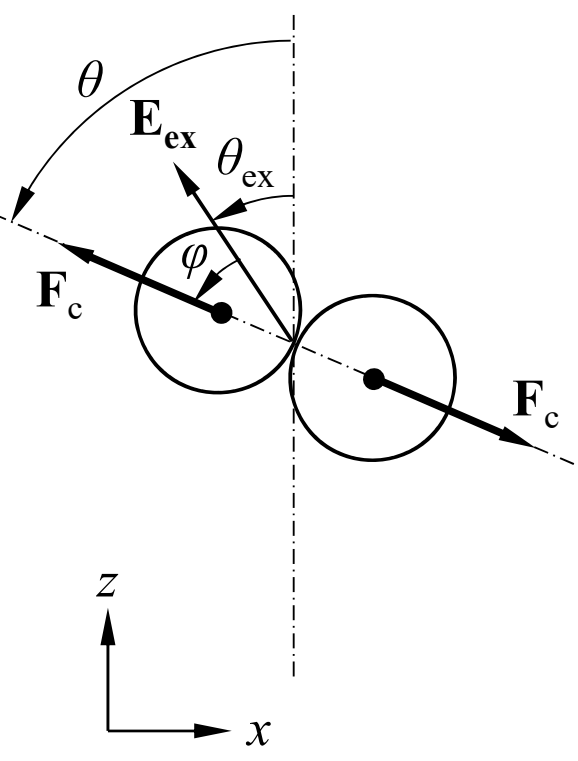

Fig. 9. Rotation models of a chain agglomerate consisting of (a) three particles and (b) two particles in an electric field. 


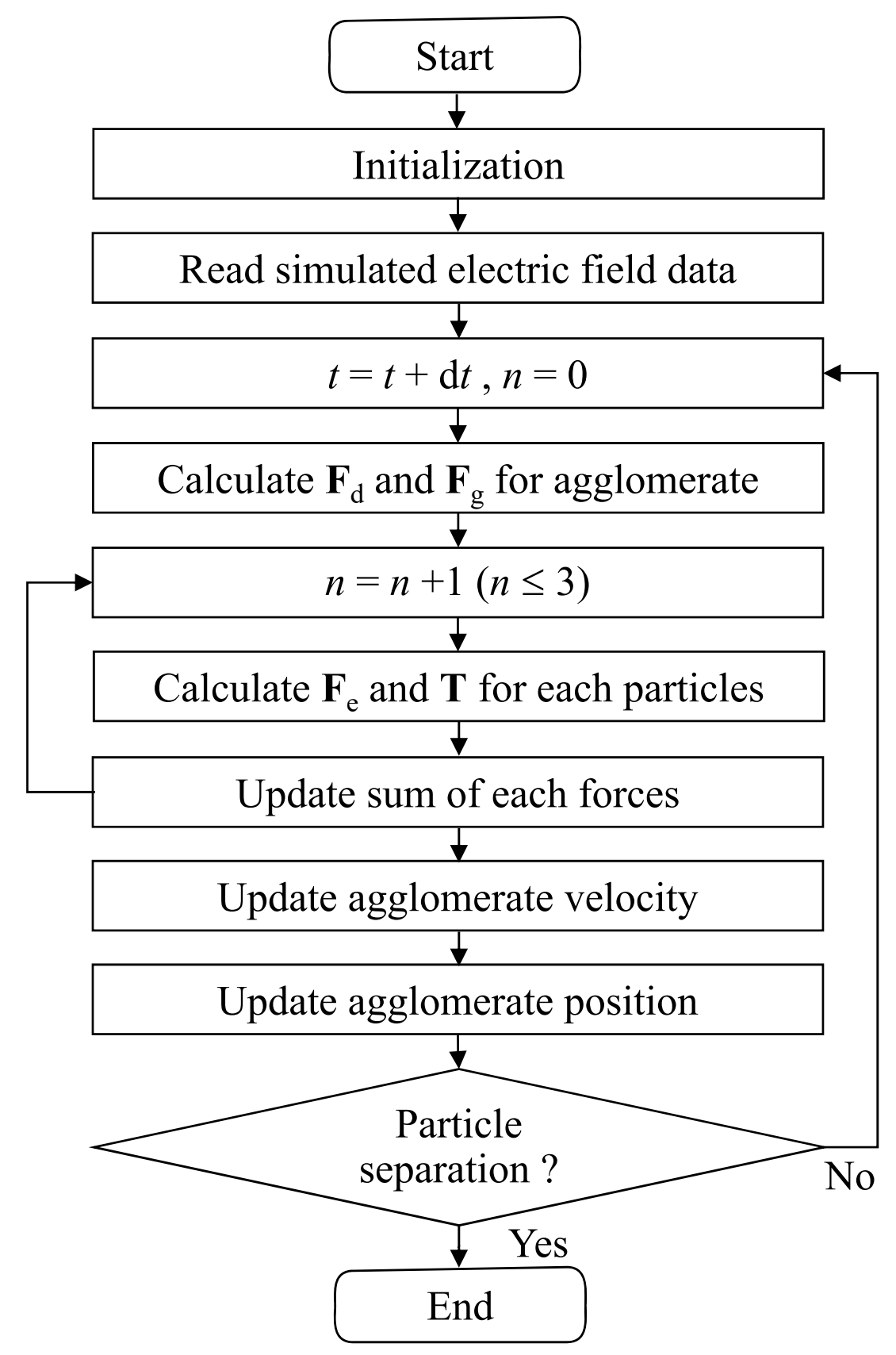

Fig. 10. Flow chart of the calculation to determine the motion of the agglomerates $(n=3)$. 


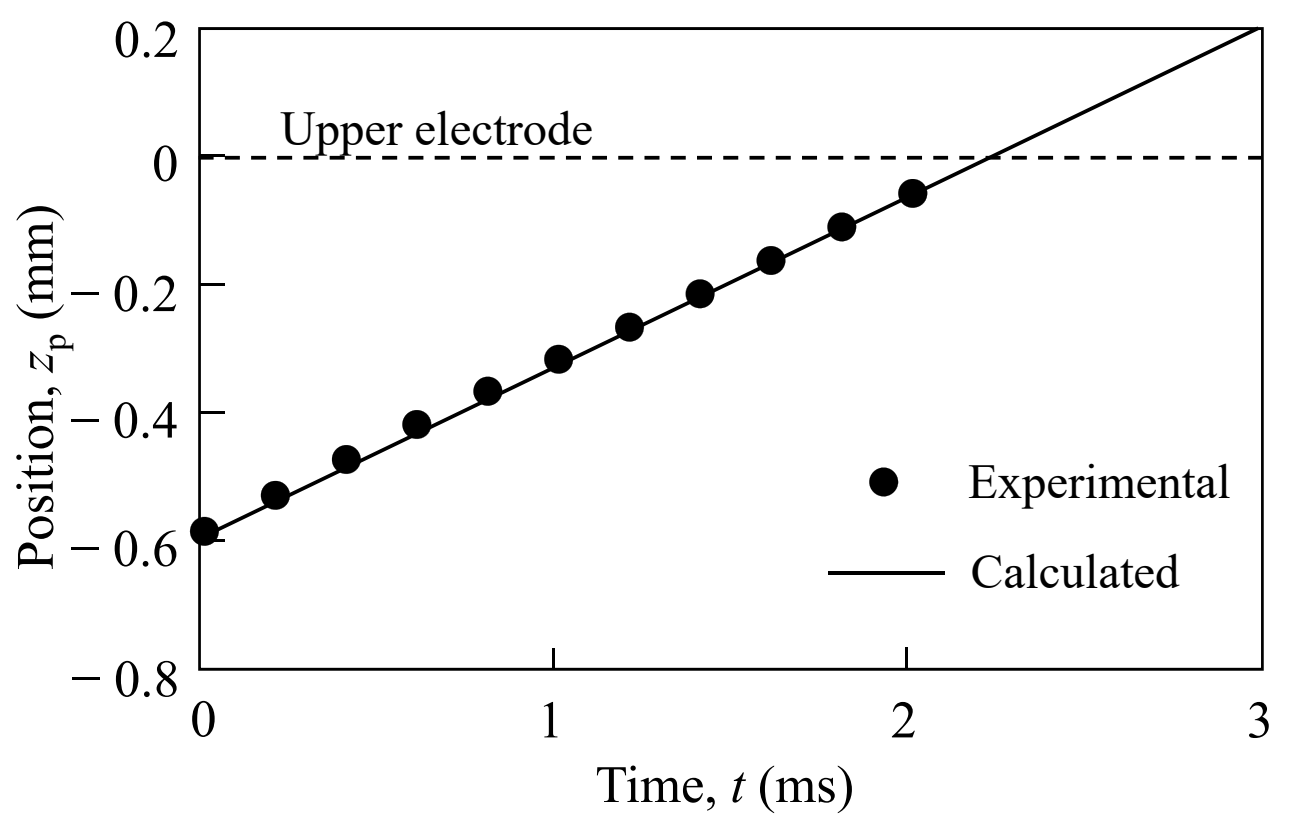

Fig. 11. Experimental and calculated vertical position of agglomerate. 


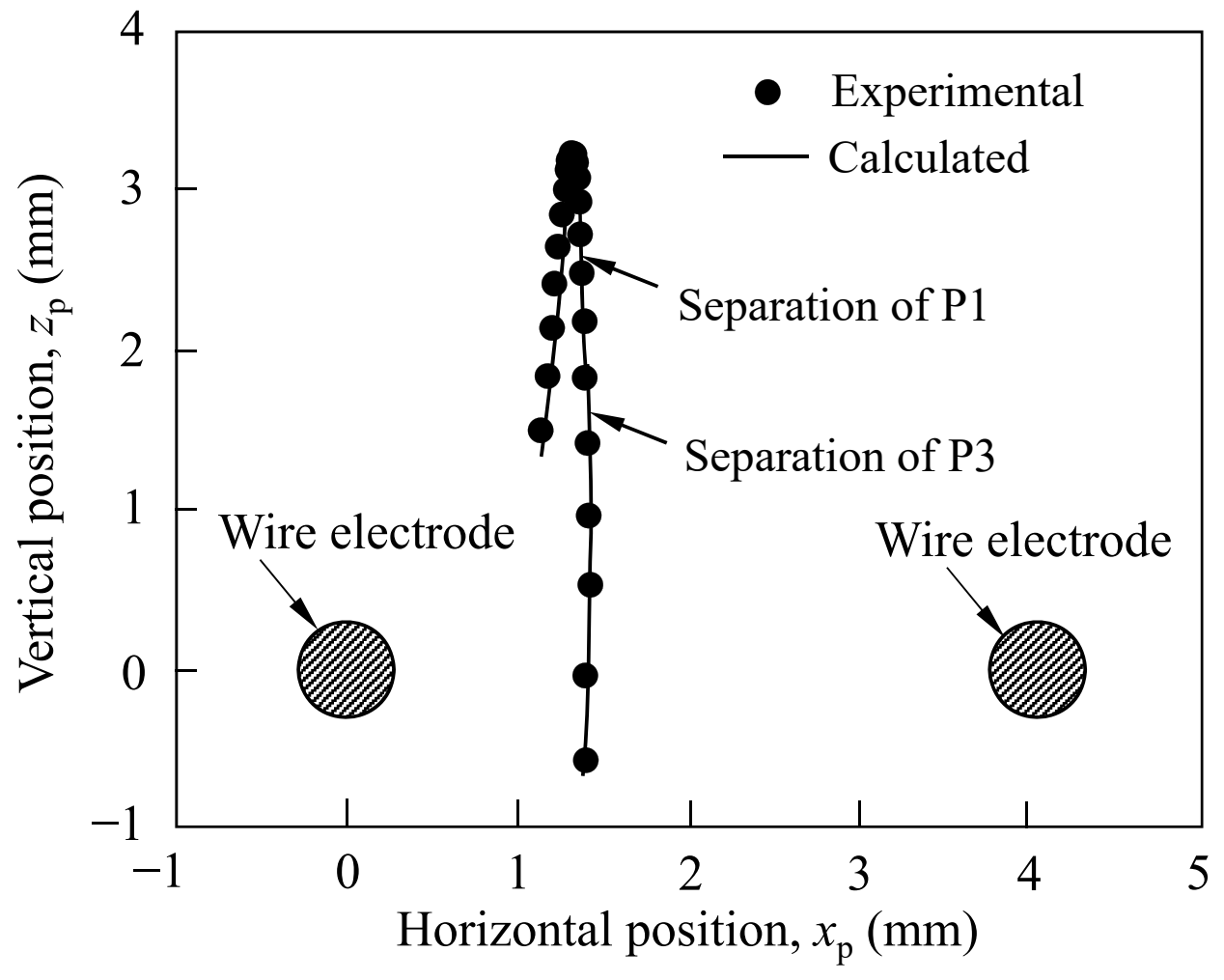

Fig. 12. Trajectory and disintegration of agglomerate with three primary particles, i.e., P1, P2, and P3. 


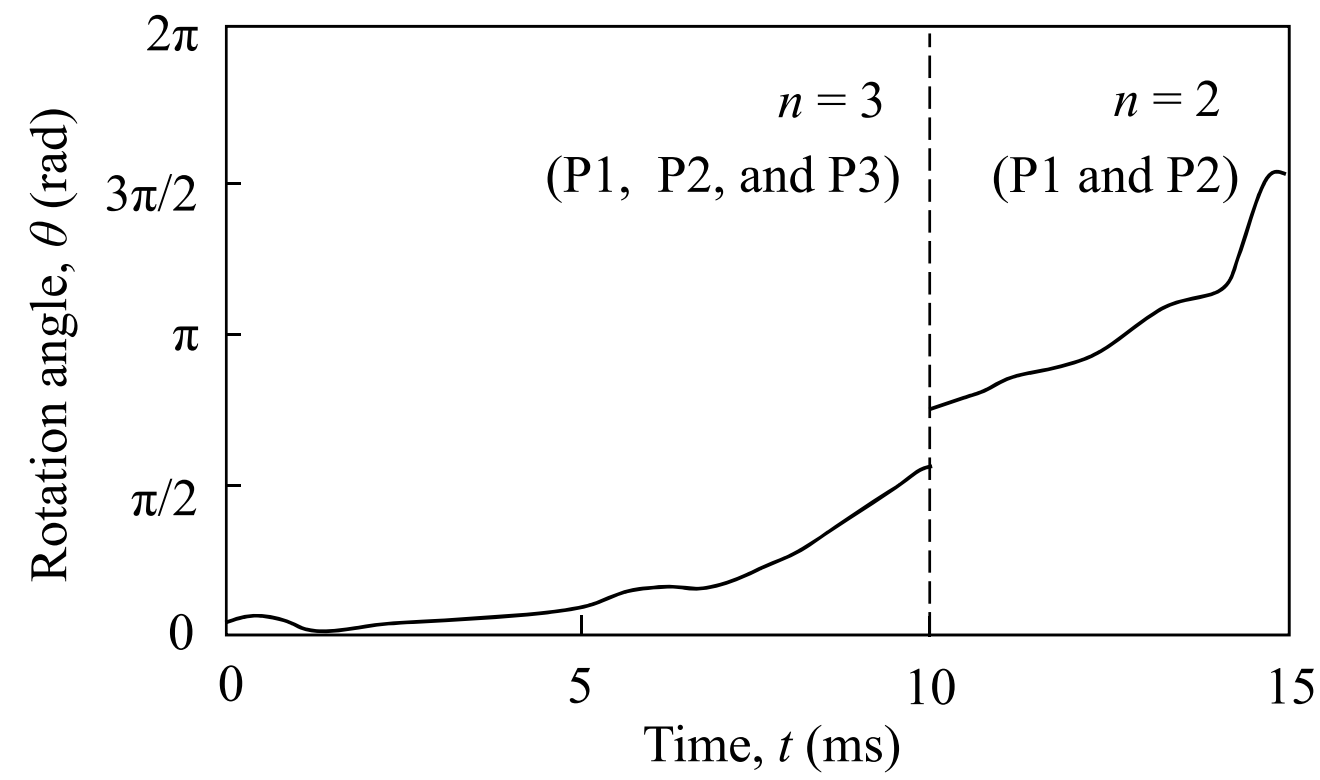

Fig. 13. Experimental result for rotation of the agglomerate. 


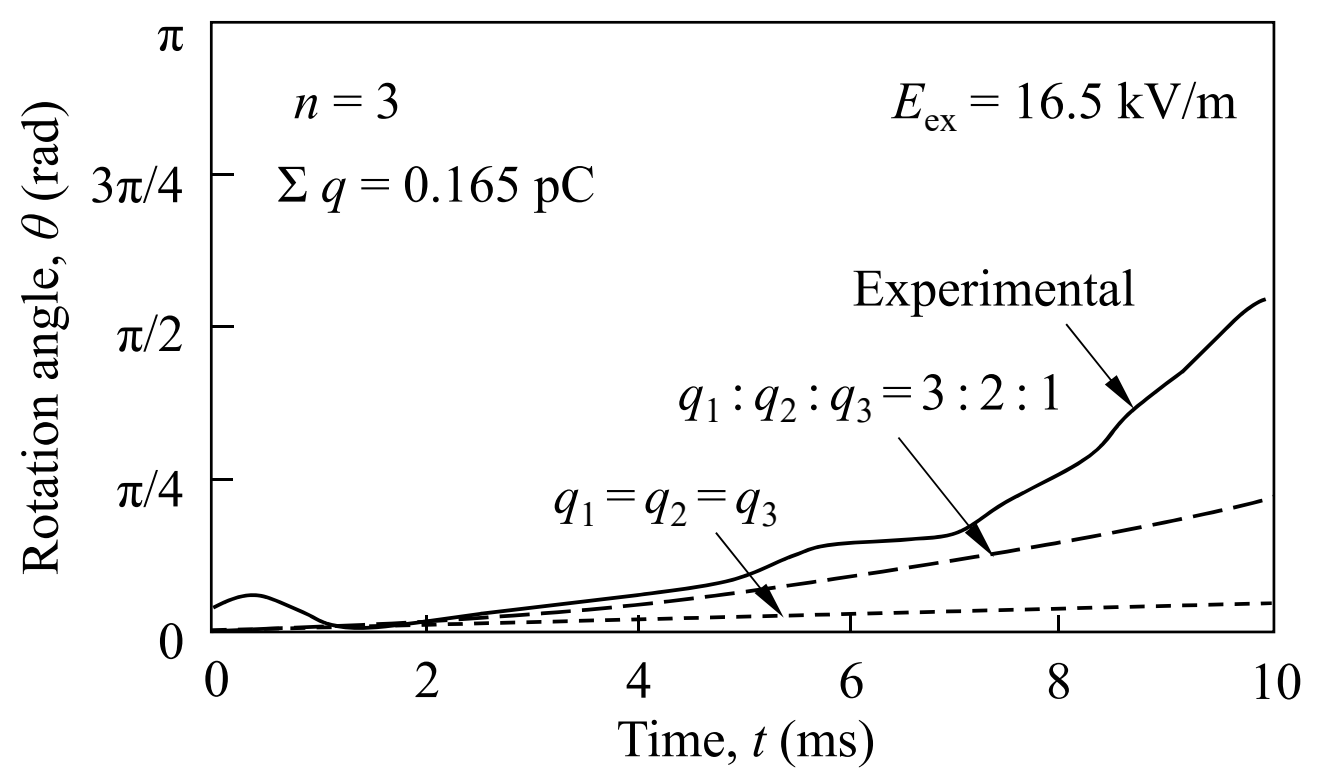

Fig. 14. Effect of particle charge distribution on rotation of the agglomerate. 

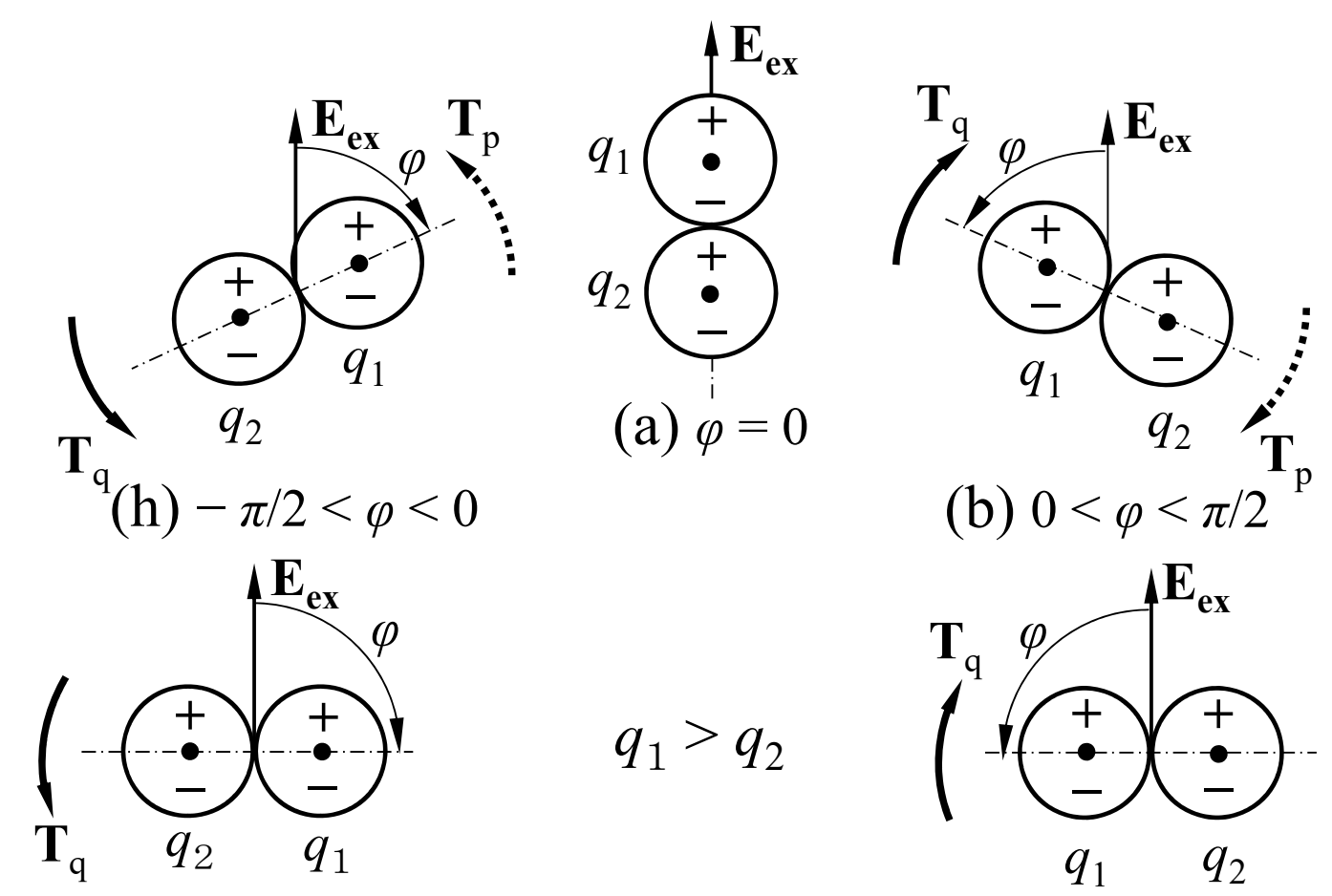

(b) $0<\varphi<\pi / 2$
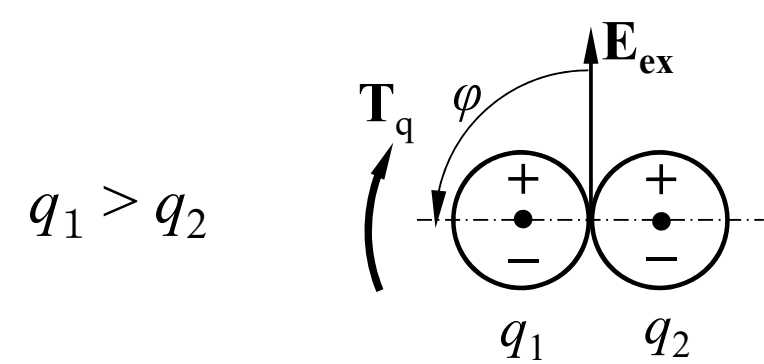

(g) $\varphi=-\pi / 2$

(c) $\varphi=\pi / 2$
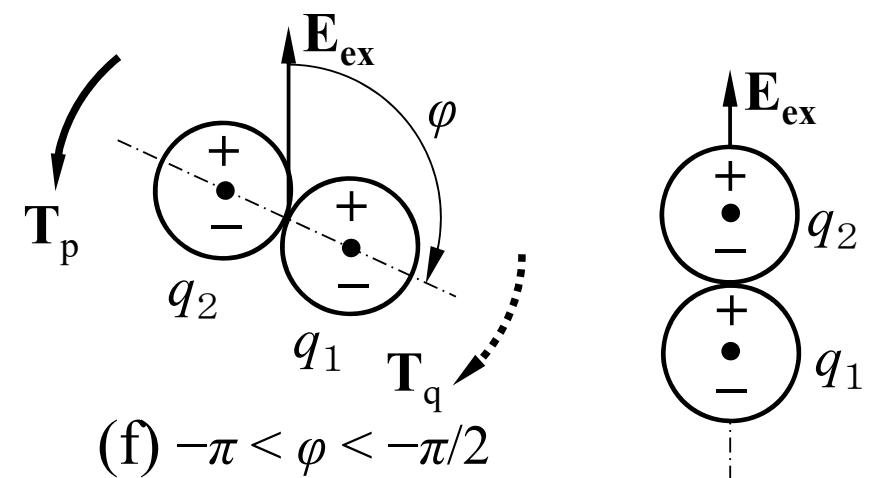

(e) $\varphi= \pm \pi$

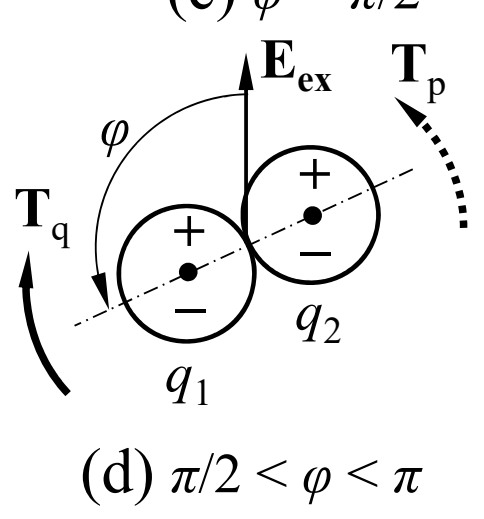

Fig. 15. Torques acting on two particles for $q_{1}>q_{2}$. 


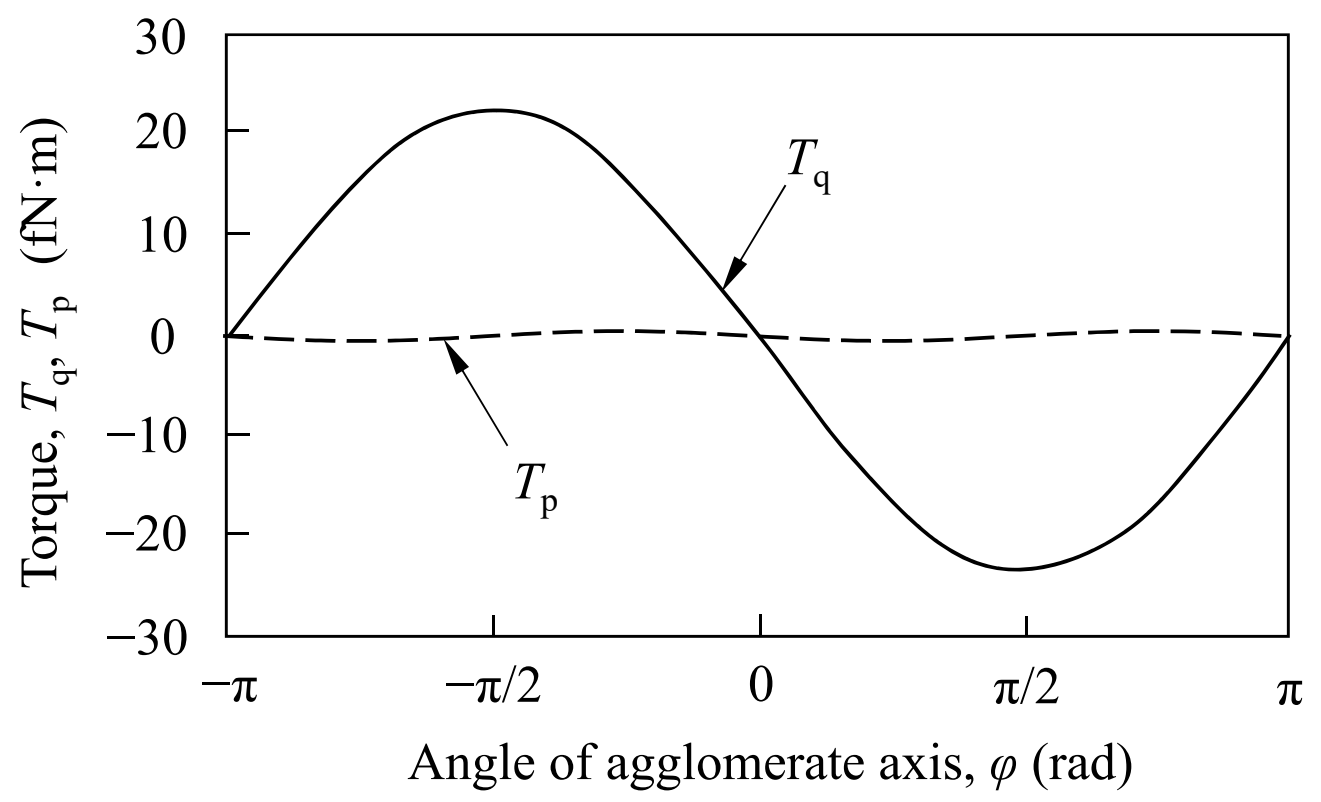

Fig. 16. Electrostatic torques calculated with $q_{1}=0.083 \mathrm{pC}, q_{2}=0.055 \mathrm{pC}$, and $E_{\mathrm{ex}}=16.5 \mathrm{kV} / \mathrm{m}$. 


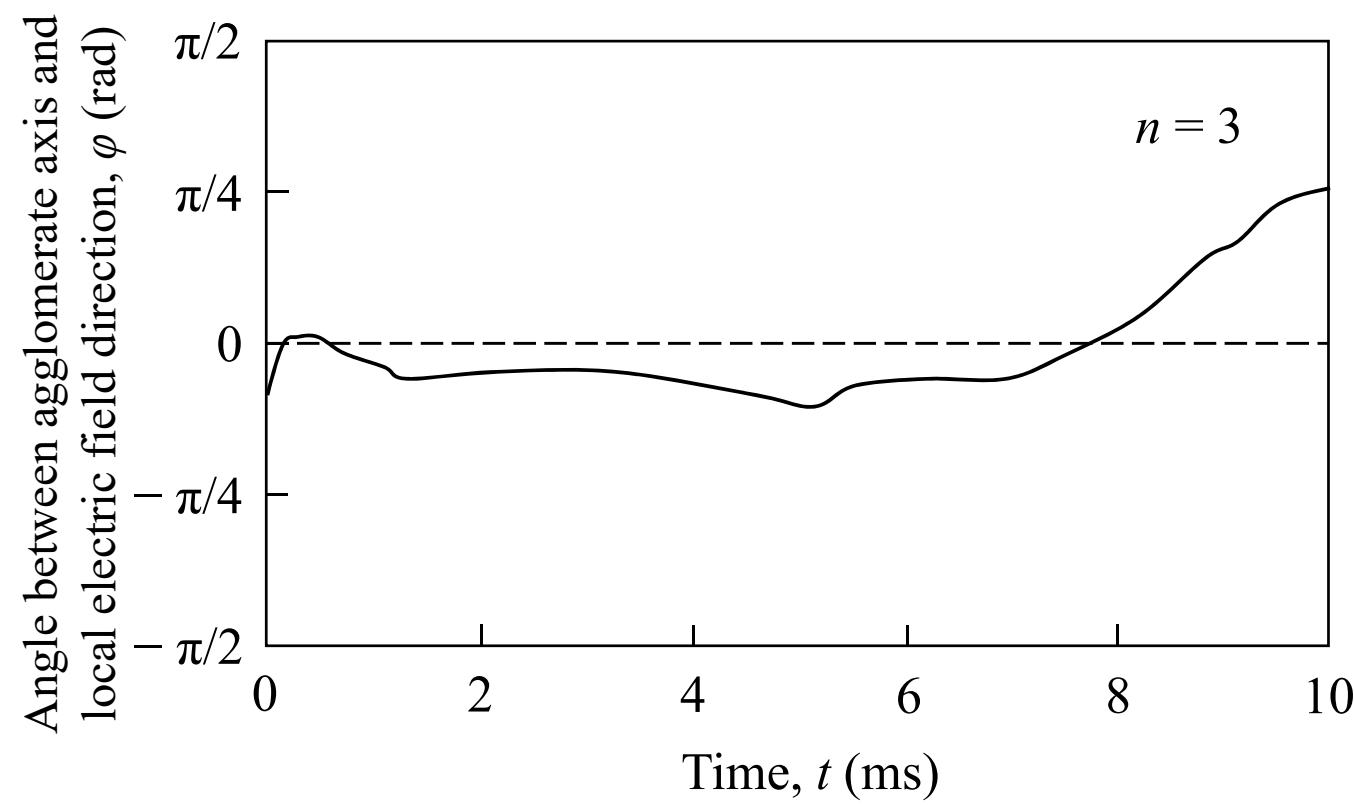

Fig. 17. Variation of angle between agglomerate axis and electric field direction. 


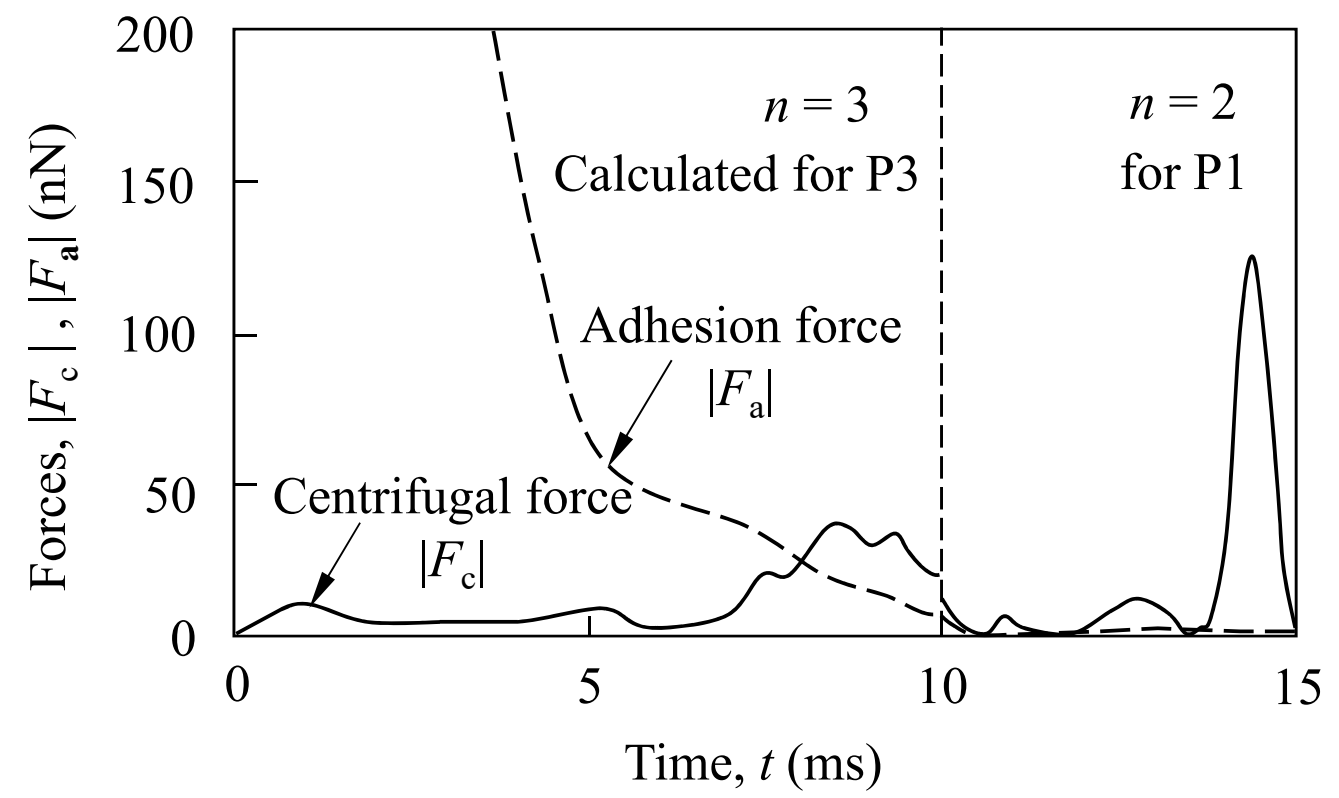

Fig. 18. Comparison between centrifugal force and adhesion force. 